\title{
Dense gas formation in the Musca filament due to the dissipation of a supersonic converging flow ${ }^{\star}$
}

\author{
L. Bonne ${ }^{1}$, N. Schneider ${ }^{2}$, S. Bontemps ${ }^{1}$, S. D. Clarke ${ }^{2}$, A. Gusdorf ${ }^{3,4}$, A. Lehmann ${ }^{3}$, M. Steinke ${ }^{2}$, T. Csengeri ${ }^{1,5}$, \\ S. Kabanovic ${ }^{2}$, R. Simon ${ }^{2}$, C. Buchbender ${ }^{2}$, and R. Güsten ${ }^{5}$ \\ ${ }^{1}$ Laboratoire d'Astrophysique de Bordeaux, Université de Bordeaux, CNRS, B18N, allée Geoffrey Saint-Hilaire, 33615 Pessac, France \\ e-mail: lars. bonne@u-bordeaux. fr \\ 2 I. Physikalisches Institut, Universität zu Köln, Zülpicher Str. 77, 50937 Köln, Germany \\ ${ }^{3}$ Laboratoire de Physique de l'École Normale Supérieure, ENS, Université PSL, CNRS Sorbonne Université de Paris, Paris, \\ France \\ ${ }^{4}$ Observatoire de Paris, PSL University, Sorbonne Université, LERMA, 75014 Paris, France \\ ${ }^{5}$ Max-Planck-Institut für Radioastronomie, Auf dem Hügel 69, 53121 Bonn, Germany
}

Received 13 November 2019 / Accepted 22 June 2020

\begin{abstract}
Observations with the Herschel Space Telescope have established that most star forming gas is organised in filaments, a finding that is supported by numerical simulations of the supersonic interstellar medium (ISM) where dense filamentary structures are ubiquitous. We aim to understand the formation of these dense structures by performing observations covering the ${ }^{12} \mathrm{CO}(4 \rightarrow 3)$, ${ }^{12} \mathrm{CO}(3 \rightarrow 2)$, and various $\mathrm{CO}(2-1)$ isotopologue lines of the Musca filament, using the APEX telescope. The observed CO intensities and line ratios cannot be explained by PDR (photodissociation region) emission because of the low ambient far-UV field that is strongly constrained by the non-detections of the [C II] line at $158 \mu \mathrm{m}$ and the [OI] line at $63 \mu \mathrm{m}$, observed with the upGREAT receiver on SOFIA, as well as a weak [CI] $609 \mu \mathrm{m}$ line detected with APEX. We propose that the observations are consistent with a scenario in which shock excitation gives rise to warm and dense gas close to the highest column density regions in the Musca filament. Using shock models, we find that the $\mathrm{CO}$ observations can be consistent with excitation by J-type low-velocity shocks. A qualitative comparison of the observed $\mathrm{CO}$ spectra with synthetic observations of dynamic filament formation simulations shows a good agreement with the signature of a filament accretion shock that forms a cold and dense filament from a converging flow. The Musca filament is thus found to be dense molecular post-shock gas. Filament accretion shocks that dissipate the supersonic kinetic energy of converging flows in the ISM may thus play a prominent role in the evolution of cold and dense filamentary structures.
\end{abstract}

Key words. ISM: individual objects: Musca - evolution - ISM: kinematics and dynamics - shock waves - turbulence stars: formation

\section{Introduction}

Observations with the Herschel Space Telescope have revealed that filamentary structures are ubiquitous in the supersonic interstellar medium (ISM; e.g. André et al. 2010; Molinari et al. 2010; Henning et al. 2010; Arzoumanian et al. 2011; Schneider et al. 2012). However, there is an ongoing discussion regarding the nature and diversity of the filaments, namely, whether they are sheets viewed edge-on or, rather, dense gas cylinders. It is also considered whether we observe a full range of filament classes: from cross-sections of sheets to dense, starforming cylindrical structures. In any case, understanding the nature, formation and evolution of filaments is essential as they are the sites of star formation. This was demonstrated by a number of recent studies which showed that pre- and protostellar cores are mostly located in filaments (e.g. Polychroni et al. 2013; André et al. 2014; Schisano et al. 2014; Könyves et al. 2015; Marsh et al. 2016; Rayner et al. 2017). In numerical simulations of the ISM, filaments are omnipresent and can form in various ways: through shocks in (magnetic) supersonic

\footnotetext{
* The reduced datacubes and images are only available at the CDS via anonymous ftp to cdsarc.u-strasbg. fr (130.79.128.5) or via http://cdsarc.u-strasbg.fr/viz-bin/cat/J/A+A/641/A17
}

turbulent colliding flows (e.g. Padoan et al. 2001; Jappsen et al. 2005; Smith et al. 2016; Federrath 2016; Clarke et al. 2017; Inoue et al. 2018), during the global gravitational collapse of a cloud (Gómez \& Vázquez-Semadeni 2014; Vázquez-Semadeni et al. 2019), through velocity shear in a magnetised medium (Hennebelle 2013), or via the gravitational instability of a sheet (Nagai et al. 1998).

However, it is challenging to find observational signatures that reveal how a filament is formed. In the view of large-scale colliding flows, filament formation is associated with the generation of warm gas from low-velocity shocks. An observational signature of these shocks are anomalously bright mid- and high$\mathrm{J}$ CO lines, that is, line integrated intensities that are higher than expected considering only heating from the far-ultraviolet (FUV) field (e.g. Pon et al. 2012). Spectroscopic Herschel observations of such lines towards the Perseus and Taurus clouds detected this excess emission (Pon et al. 2014; Larson et al. 2015), where it was proposed to be the result of low-velocity shocks $\left(v_{\text {shock }}<\right.$ $4 \mathrm{~km} \mathrm{~s}^{-1}$ ), dissipating the overall, generic supersonic turbulence of a molecular cloud.

In this paper, we report on the excess emission seen in mid$\mathrm{J}{ }^{12} \mathrm{CO}$ lines observed around the Musca filament and propose that in low- to moderate density regions, exposed to a very weak 


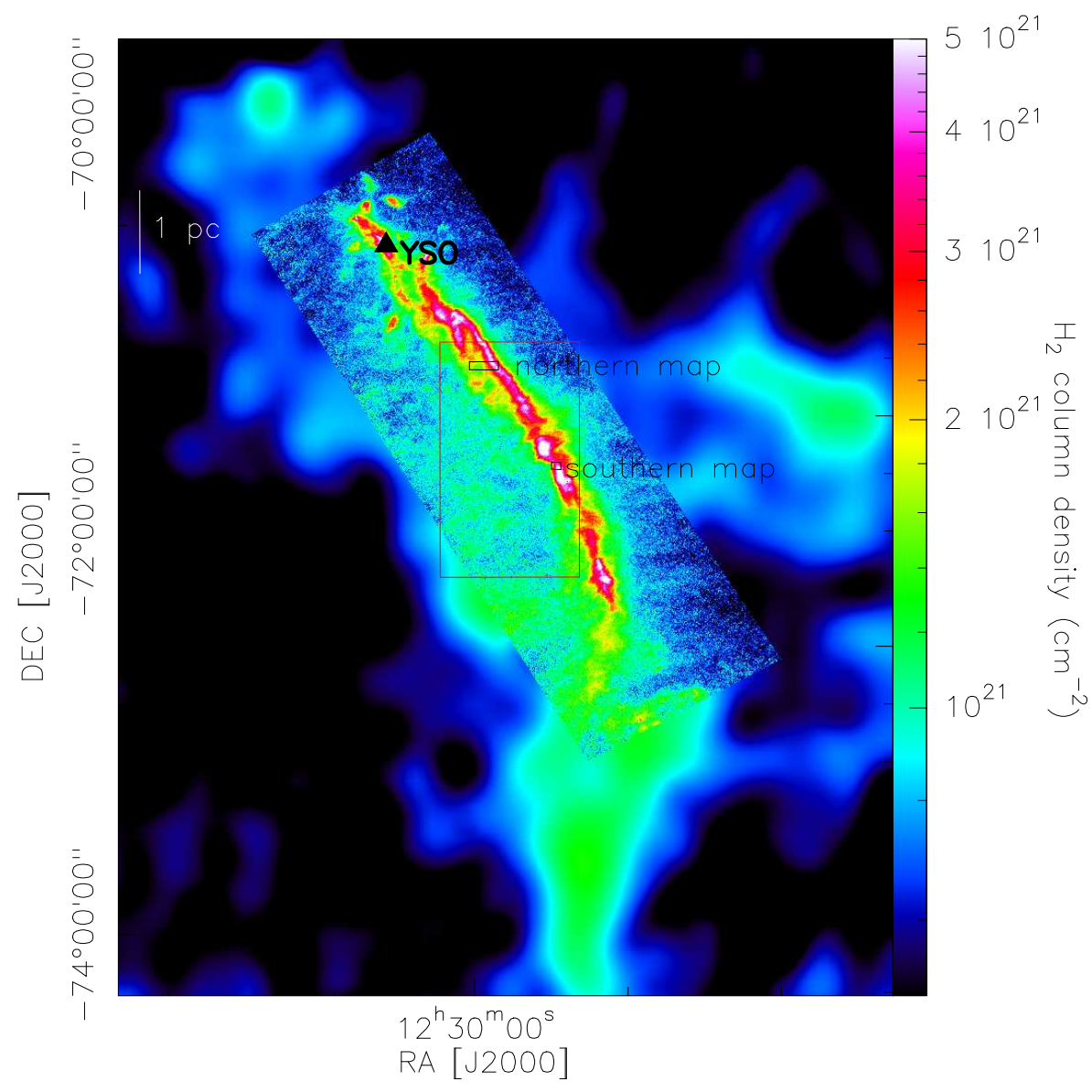

Fig. 1. Herschel column density map of the Musca filament (Cox et al. 2016) inserted into the extinction map of the Musca cloud (Schneider et al. 2011), which is scaled to the Herschel column density map. The filament crest is defined as $N>3 \times 10^{21} \mathrm{~cm}^{-2}$ (purple and white). The region outlined by a red rectangle is the zoom displayed in Fig. 2. The ambient cloud is displayed in green and blue. The northern and southern areas mapped with FLASH+ on the APEX telescope are indicated in black. The triangle indicates the location of the only YSO in the Musca filament.

FUV field, these lines may also serve as a tracer for low-velocity shocks. The Musca filament is located at a distance of $140 \mathrm{pc}$ (Franco 1991). We adopt the nomenclature from Cox et al. (2016) for the different features of the Musca filament and we indicate them in Fig. 2 (see also Fig. 2 in Cox et al. 2016). First, there is the high column density filament crest with $N>3 \times 10^{21} \mathrm{~cm}^{-2}$ (similar value to the one used by Cox et al. (2016) which is $N>2.7 \times 10^{21} \mathrm{~cm}^{-2}$ ). In addition, $\mathrm{C}^{18} \mathrm{O}(2-1)$ emission basically disappears below this column density value. Attached to the filament crest are intermediate column density $\left(N \sim 2 \times 10^{21} \mathrm{~cm}^{-2}\right)$ hair-like structures called strands, with a size of $\sim 0.2-0.4 \mathrm{pc}$. Even further outwards at larger distances, narrow straight structures of up to a few parsec lengths, called striations (Goldsmith et al. 2008; Palmeirim et al. 2013; Alves de Oliveira et al. 2014; Heyer et al. 2016; Cox et al. 2016; Malinen et al. 2016) are seen. They are mostly orthogonal to the crest and located in the ambient cloud. The ambient cloud then refers to the environmental gas, traced by the extinction map in Fig. 1, embedding the denser Musca filament crest and strands. The filament crest is velocitycoherent (Hacar et al. 2016) and has only one young stellar object (YSO) located at the northern end (e.g. Vilas-Boas et al. 1994, Fig. 1). It was recently proposed that Musca is a sheet seen edgeon (Tritsis \& Tassis 2018). However, one of the main results of a companion paper (Bonne et al., in prep., hereafter Paper I), is that the Musca filament crest is more consistent with a cylindrical geometry at a density of $n_{\mathrm{H}_{2}} \sim 10^{4} \mathrm{~cm}^{-3}$. Because other authors (Cox et al. 2016; Kainulainen et al. 2016; Hacar et al. 2016) also support this cylindrical geometry, we adopt this view as a working hypothesis.

In Paper I, the large scale kinematics and physical conditions in the Musca filament and cloud are studied with the main

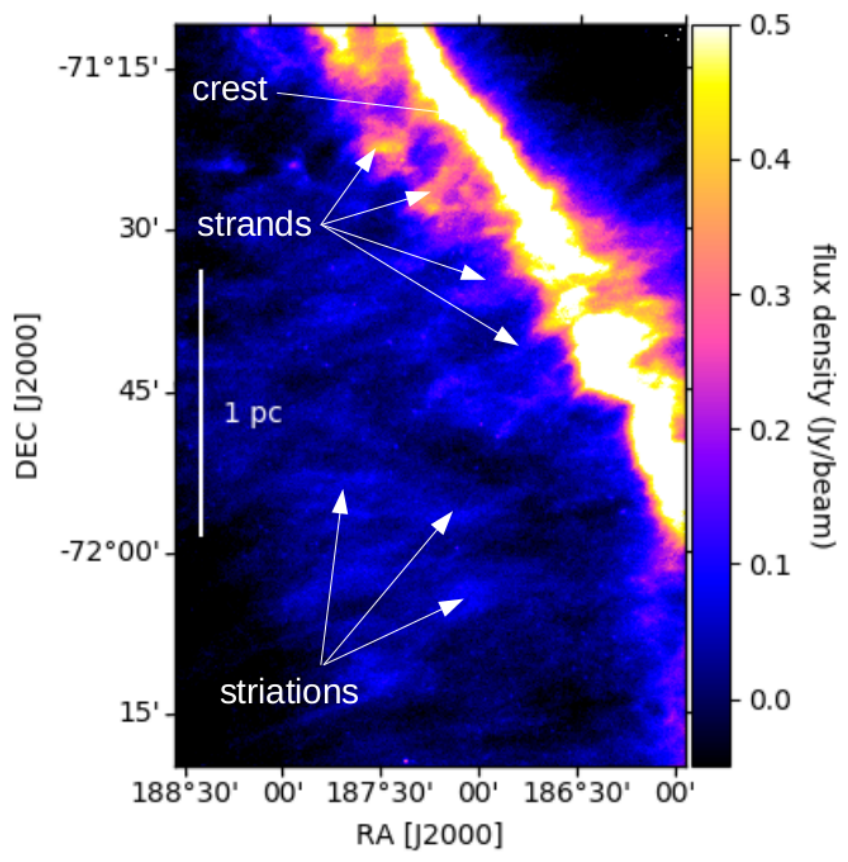

Fig. 2. Zoom (red box in Fig. 1) into the Herschel $250 \mu \mathrm{m}$ map of Musca, indicating the striations, strands and the filament crest (Cox et al. 2016).

$\mathrm{CO}(2-1)$ isotopologues, as well as the relation between Musca and the Chamaeleon-Musca complex. In this paper, we present ${ }^{12} \mathrm{CO}(4-3)$ and ${ }^{12} \mathrm{CO}(3-2)$ lines and argue for the presence of 
a blueshifted excess velocity component best visible towards the filament crest and strands. A detailed analysis of this 'blueshifted component' is the objective of this paper.

Here, we present a non-LTE CO excitation analysis of the blueshifted component, as well as complementary observations of the far-infrared fine structure transitions of carbon ([C $\mathrm{C}])$, oxygen $\left(\left[\mathrm{O}_{\mathrm{I}}\right]\right)$ and ionised carbon $([\mathrm{C} \mathrm{II}])$, which reveals a warm gas component around the filament. We show that the excitation of this warm gas can be explained by a filament accretion shock, that is, a low-velocity shock due to mass accretion on a filament from a converging flow in an interstellar cloud.

\section{Observations}

\subsection{APEX}

We used the FLASH460 receiver on the APEX telescope (Güsten et al. 2006; Klein et al. 2014), and obtained a single pointing in $\left[\mathrm{C}_{\mathrm{I}}\right]{ }^{3} \mathrm{P}_{1}-{ }^{3} \mathrm{P}_{0}$ at $609 \mu \mathrm{m}$ towards the Musca filament at $\alpha_{2000}=12^{\mathrm{h}} 28^{\mathrm{m}} 55^{\mathrm{s}}$ and $\delta_{2000}=-71^{\circ} 16^{\prime} 55^{\prime \prime}$, using the OFF position $\alpha_{2000}=12^{\mathrm{h}} 41^{\mathrm{m}} 38^{\mathrm{s}}$ and $\delta_{2000}=-71^{\circ} 11^{\prime} 00^{\prime \prime}$ (Hacar et al. 2016). The beamsize is $13^{\prime \prime}$ and the spectral resolution is $\sim 0.05 \mathrm{~km} \mathrm{~s}^{-1}$. The data reduction was done with the CLASS software $^{1}$. A main beam efficiency ${ }^{2} \eta_{\mathrm{mb}}=0.49$ was applied for $\left[\mathrm{C}_{\mathrm{I}}\right]$, a baseline of order one was removed, and a correction for a $490 \mathrm{kHz}$ shift in the FLASH460 instrument was performed (see Paper I, F. Wyrowski, priv. comm.). Fitting the [ $\left.\mathrm{C}_{\mathrm{I}}\right]$ single pointing (Fig. 3) with a Gaussian profile provides a peak temperature brightness $T_{\mathrm{mb}}=3.5 \mathrm{~K}$ and $F W H M=0.8 \mathrm{~km} \mathrm{~s}^{-1}$ (or $3.4 \times 10^{-7} \mathrm{erg} \mathrm{s}^{-1} \mathrm{~cm}^{-2} \mathrm{sr}^{-1}$ ).

The CO observations with APEX are presented in more detail in Paper I, but here we shortly summarise the most important facts. All data was obtained in 2017 and 2018, using FLASH345 and FLASH460 for the ${ }^{12} \mathrm{CO}(3-2)$ and ${ }^{12} \mathrm{CO}(4-3)$ mapping of the northern and southern regions (Fig. 1). The FLASH345 (FLASH460) observations have a spectral resolution of $0.033(0.05) \mathrm{km} \mathrm{s}^{-1}$ and an angular resolution of $18^{\prime \prime}\left(14^{\prime \prime}\right)$. Main beam efficiencies of $\eta_{\mathrm{mb}}=0.65(0.49)$ were applied to the antenna temperatures. We here use data sampled to $0.1 \mathrm{~km} \mathrm{~s}^{-1}$ and smoothed to a resolution of $28^{\prime \prime}$. This allows a better comparison to the $\mathrm{CO}(2-1)$ APEX data which were taken with the PI230 receiver. The spectral resolution for $\mathrm{CO}(2-1)$ is $0.08 \mathrm{~km} \mathrm{~s}^{-1}$ at an angular resolution of $28^{\prime \prime}$. Here, we applied a main beam efficiency of $\eta_{\mathrm{mb}}=0.68$. The center $(0,0)$ position of the maps is $\alpha_{2000}=12^{\mathrm{h}} 28^{\mathrm{m}} 58^{\mathrm{s}}, \delta_{2000}=-71^{\circ} 16^{\prime} 55^{\prime \prime}$ for the northern map and $\alpha_{2000}=12^{\mathrm{h}} 24^{\mathrm{m}} 46^{\mathrm{s}}, \delta_{2000}=-71^{\circ} 47^{\prime} 20^{\prime \prime}$ for the southern map.

\subsection{SOFIA}

In June 2018, the upGREAT instrument (Risacher et al. 2018) on the Stratopsheric Observatory for Far-Infrared Astronomy (SOFIA) was used for a 70 min single pointing of the 7 pixel array covering the atomic oxygen $\left[\mathrm{O}_{\mathrm{I}}\right]{ }^{3} \mathrm{P}_{1}-{ }^{3} \mathrm{P}_{2}$ fine structure line at $63 \mu \mathrm{m}$ and the ionised carbon $\left[\mathrm{C}_{\mathrm{II}}\right]{ }^{2} \mathrm{P}_{3 / 2}{ }^{2} \mathrm{P}_{1 / 2}$ fine structure line at $158 \mu \mathrm{m}$ towards the southern APEX map at $\alpha_{2000}=$ $12^{\mathrm{h}} 24^{\mathrm{m}} 41^{\mathrm{s}} .6, \delta_{2000}=-71^{\circ} 46^{\prime} 41^{\prime \prime} \cdot 0$. These observations were performed in the single beam switching mode with a chop amplitude of $150^{\prime \prime}$ towards a location with weak or no emission in all Herschel far-infrared bands $(70-500 \mu \mathrm{m})$. This was done to obtain a good baseline for such sensitive observations. Mars was

\footnotetext{
1 http://wWw . iram. fr/IRAMFR/GILDAS

2 http://www . apex-telescope.org/telescope/efficiency/
}
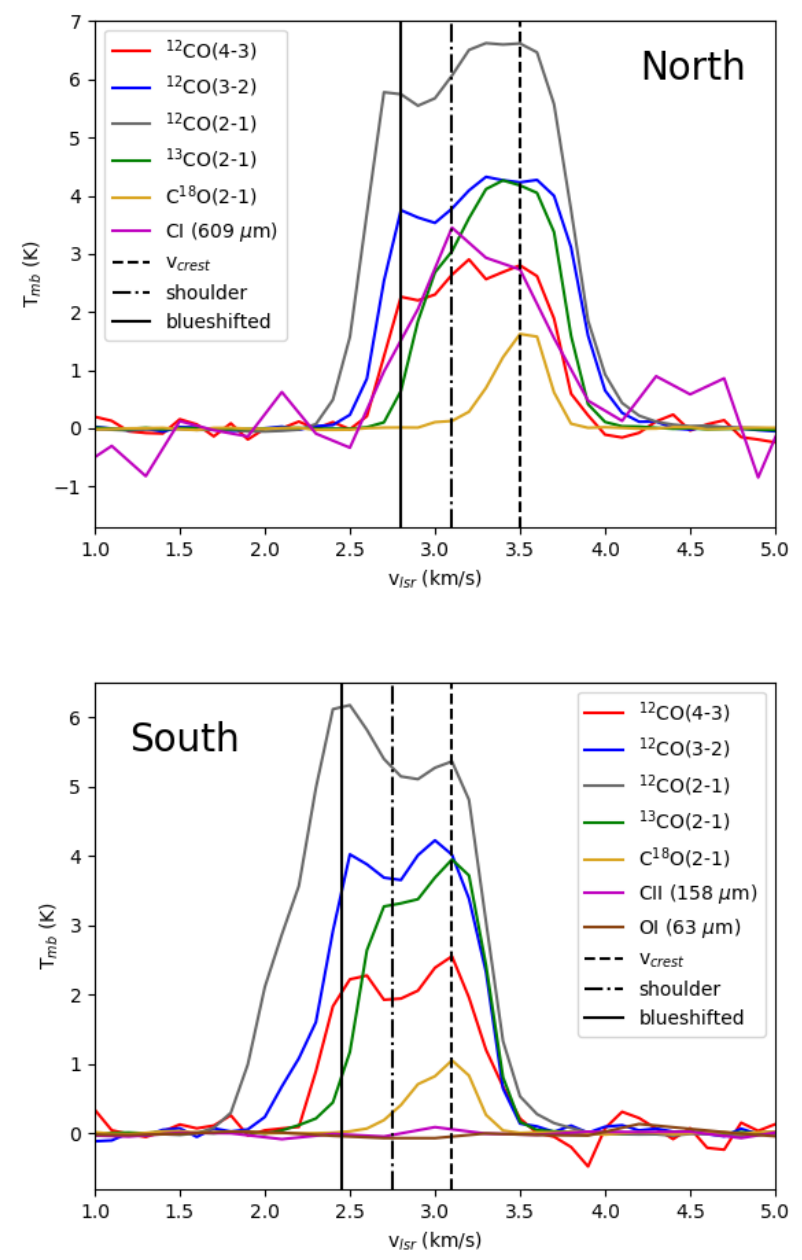

Fig. 3. Top: APEX CO and [CI] spectra in the northern map, averaged over the filament crest $\left(N>3 \times 10^{21} \mathrm{~cm}^{-2}\right)$. Bottom: APEX CO spectra averaged over the southern map and the 7-pixel averaged SOFIA [C II] and [O I] lines that are not detected above the $3 \sigma$ level. We note the prominent blueshifted velocity component around $2.8 \mathrm{~km} \mathrm{~s}^{-1}$ in the north and at $2.5 \mathrm{~km} \mathrm{~s}^{-1}$ in the south, only visible in the ${ }^{12} \mathrm{CO}$ lines.

used as a calibrator to determine the main beam efficiencies of the individual pixels. The fitted water vapor column was typically around $10 \mu \mathrm{m}$. All the intensities reported here are on the main beam temperature scale. The $\left[\mathrm{O}_{\mathrm{I}}\right]$ and $[\mathrm{C} \mathrm{II}$ observations have a main beam size of $6.3^{\prime \prime}$ and $14.1^{\prime \prime}$, respectively, and both data sets were smoothed to a spectral resolution of $0.3 \mathrm{~km} \mathrm{~s}^{-1}$.

There is no detection of the $[\mathrm{CII}]$ and $[\mathrm{OI}]$ lines (Appendix A) in the individual pixels (Fig. A.1) or in the arrayaveraged spectra (Fig. 3). The $3 \sigma$ upper limits for the two lines are 0.11 and $0.19 \mathrm{~K}$, equivalent to $7.5 \times 10^{-7} \mathrm{erg} \mathrm{s}^{-1} \mathrm{~cm}^{-2} \mathrm{sr}^{-1}$ and $2.1 \times 10^{-5} \mathrm{erg} \mathrm{s}^{-1} \mathrm{~cm}^{-2} \mathrm{sr}^{-1}$ when assuming a FWHM of $1 \mathrm{~km} \mathrm{~s}^{-1}$, for [ $\left.\mathrm{C}_{\mathrm{II}}\right]$ and $\left[\mathrm{O}_{\mathrm{I}}\right]$, respectively.

\section{Results and analysis}

\subsection{The ambient FUV field from a census of nearby stars}

We estimated the FUV field upper limit in Musca using a census of nearby ionising stars (e.g. Schneider et al. 2016). For that, we employed the SIMBAD ${ }^{3}$ database (Wenger et al. 2000) and

\footnotetext{
3 http://simbad.u-strasbg.fr/simbad/sim-fsam
} 
imposed a search for B3 and earlier type stars within a radius of $40^{\circ}(100 \mathrm{pc})$, centered on the Musca filament, and a maximal distance of $250 \mathrm{pc}$. This provided a census of 59 ionising stars including two dominant O9-type stars. Knowing the spectral type of these ionising stars, we estimated the contribution of the FUV emission using model atmospheres ${ }^{4}$ of Kurucz (1979) with solar abundances (Grevesse \& Sauval 1998) and $\log (g) \sim 4.0(g$ is the surface gravity). The temperature of the different spectral types was obtained from Fitzpatrick \& Massa (2005) and Pecaut \& Mamajek (2013). The distance information and the location on the sky of both Musca and the stars then allows to calculate their relative distances. We assumed a $r^{-2}$ decrease of the flux and projected all stars in the plane of the sky, and obtained in this way an ambient FUV field of $3.4 G_{0}$ (Habing 1968) for the Musca cloud as an upper limit. The real field is lower because we did not take into account any extinction, that is, attenuation by diffuse gas and blocking by molecular clumps.

\subsection{Limits on FUV heating from $\mathrm{Cl} \& \mathrm{Cll}$}

As a second method to constrain the ambient FUV field, we used the SOFIA [ $\left.\mathrm{C}_{\mathrm{II}}\right]$ upper limit and the APEX $\left[\mathrm{C}_{\mathrm{I}}\right]$ brightness observed in Musca. [C $\mathrm{II}]$ is an excellent tracer of the FUV field at low densities as it is a direct result of the ionising radiation (e.g. Tielens \& Hollenbach 1985) and its non-detection points towards a low value for the ambient FUV field. $\left[\mathrm{C}_{\mathrm{I}}\right]$ is not a direct product of FUV ionisation, but PDR models demonstrate that its brightness is also sensitive to the FUV field (e.g. Hollenbach \& Tielens 1997; Röllig et al. 2007). In this section, we compare the observed line brightness with predictions from PDR models using the 2006 models of the PDR toolbox ${ }^{5}$ (Kaufman et al. 2006; Pound \& Wolfire 2008). These plots are expressed in molecular hydrogen density $n_{\mathrm{H}_{2}}$, where we assume that all hydrogen atoms are locked in molecular hydrogen.

The [C II] upper limit restricts the FUV field to values $<1 G_{0}$ for densities $n_{\mathrm{H}_{2}} \leq 10^{4} \mathrm{~cm}^{-3}$. This is consistent with the intensity of the $\left[\mathrm{C}_{\mathrm{I}}\right]$ line which is so weak that it is below the minimum value in the PDR toolbox. Even with a beam filling value of $30 \%$ for $\left[\mathrm{C}_{\mathrm{I}}\right]$, which would be low around the Musca filament, the FUV field remains below $1 G_{0}$ for typical densities of the Musca ambient cloud. Using the Meudon PDR $\operatorname{code}^{6}$ (Le Petit et al. 2006; Le Bourlot et al. 2012; Bron et al. 2014) for the same lines, confirms that the upper limit on the $\left[\mathrm{C}_{\mathrm{II}}\right]$ intensity can only be the result of a FUV field strength $<1 G_{0}$. With these observational upper limits for the ambient FUV field, the Kaufman et al. (2006) PDR models restrict the maximal surface temperature of the cloud to $25 \mathrm{~K}$. This temperature upper limit for gas embedded in such a weak FUV field is also found in other theoretical models where heating by the FUV field is taken into account (e.g. Godard et al. 2019), and consistent with the maximal CO temperature in numerical simulations of molecular clouds evolving in a FUV field $\leq 1.7 G_{0}$ (Glover \& Smith 2016; Clark et al. 2019).

\subsection{CO spectra in Musca}

\subsubsection{Three $\mathrm{CO}$ velocity components}

Figure 3 shows spectra averaged across the northern (top panel) and southern (bottom panel) filament for Herschel column densities $\mathrm{N}>3 \times 10^{21} \mathrm{~cm}^{-2}$.

\footnotetext{
4 http://www.oact.inaf.it/castelli/castelli/grids.html

5 http://dustem.astro.umd.edu/

6 http://ismdb.obspm.fr/
}

The spectra of the CO isotopologues unveil three velocity components that show small velocity variations in the north and south. First, there is a single component in $\mathrm{C}^{18} \mathrm{O}(2-1)$ at $3.5 \mathrm{~km} \mathrm{~s}^{-1}$ (north) and $3.1 \mathrm{~km} \mathrm{~s}^{-1}$ (south) that traces the Musca filament crest (Hacar et al. 2016). In ${ }^{13} \mathrm{CO}(2-1)$ one observes a blueshifted shoulder to the velocity component of the filament crest, which is a velocity component related to the strands, namely, the dense interface region between the filament crest and the ambient cloud. It has a typical velocity of $3.1 \mathrm{~km} \mathrm{~s}^{-1}$ in the north and $2.7 \mathrm{~km} \mathrm{~s}^{-1}$ in the south and is not detected in $\mathrm{C}^{18} \mathrm{O}$ with current data. In ${ }^{12} \mathrm{CO}$ a third velocity component is observed, without any clearly detected counterpart in ${ }^{13} \mathrm{CO}$ or $\mathrm{C}^{18} \mathrm{O}$, which is further blueshifted so that we call it the "blueshifted component". The brightness peak of the blueshifted component in the northern map occurs at $2.8 \mathrm{~km} \mathrm{~s}^{-1}$ and in the southern map at $2.5 \mathrm{~km} \mathrm{~s}^{-1}$. This blueshifted component is the focus of this paper.

The individual ${ }^{12} \mathrm{CO}(4-3)$ spectra in the northern map are presented in Fig. 4, overlaid on the Herschel column density and temperature map in the top panels and together with individual spectra in other $\mathrm{CO}$ isotopologues at selected positions in the bottom panel. Inspecting the ${ }^{12} \mathrm{CO}(3-2)$ and ${ }^{12} \mathrm{CO}(4-3)$ spectra, we find that these lines are clearly detected towards the lower column density regions and that this blueshifted component is present over the entire map, see Fig. 4.

Because our assumption is that the Musca filament is cylindrical, the observed $\mathrm{CO}$ emission in direction of the crest and strands also contains gas from the ambient cloud along the lineof-sight. In particular, the ${ }^{12} \mathrm{CO}(2-1)$ blueshifted line can have a more significant component arising from this gas phase. We can thus not fully exclude effects of high optical depth and selfabsorption, so we run tests using a two-layer gas model for the $\mathrm{CO}(2-1)$ isotopologue lines in order to calculate a possible impact of foreground absorption and present the results in Appendix B. For that, we assumed only 2 CO line components, i.e. the crest component and a shoulder+blueshifted one that it self-absorbed and only apparently shows separate components. However, the result of this modelling is that it is not possible to reproduce the observed $\mathrm{CO}$ line profiles with only two components, so that we are confident in our approach to use 3 separate lines. The best fitting model for the blueshifted component of all CO lines in the northern and southern map is the one with the parameters given in Table 1 . We note from the Table that the fitted ${ }^{12} \mathrm{CO}(2-1)$ linewidth of the blueshifted component in the south is higher than the linewidth obtained for ${ }^{12} \mathrm{CO}(3-2)$ and ${ }^{12} \mathrm{CO}(4-3)$, which have a similar width. It is thus possible that there, the low-J ${ }^{12} \mathrm{CO}(2-1)$ line traces more material along the line-of-sight from the ambient cloud. Inspecting the spectra, see Fig. 3, confirms that ${ }^{12} \mathrm{CO}(3-2)$ and ${ }^{12} \mathrm{CO}(4-3)$ only show up at higher velocities and have a smaller linewidth. This difference, and the irregular ${ }^{12} \mathrm{CO}(2-1)$ shape in the southern map, suggests that ${ }^{12} \mathrm{CO}(2-1)$ traces the ambient cloud down to lower velocities than the detected ${ }^{12} \mathrm{CO}(4-3)$ and (3-2) emission. We also determined from the averaged ${ }^{12} \mathrm{CO}(2-1)$ and ${ }^{13} \mathrm{CO}(2-1)$ spectra that the ${ }^{12} \mathrm{CO}(2-1) /{ }^{13} \mathrm{CO}(2-1)$ line ratio in the blueshifted component in the northern and southern map are between 15 and 60 , which are expected values for optically thin emission. Note that fractionation (see Appendix B) can cause observed values for the ${ }^{12} \mathrm{CO} /{ }^{13} \mathrm{CO}$ ratio significantly below 60 . This is in agreement with early indications for Musca of significant $\mathrm{CO}$ isotopologue abundace variations (Hacar et al. 2016) which we further confirm in Paper I. 

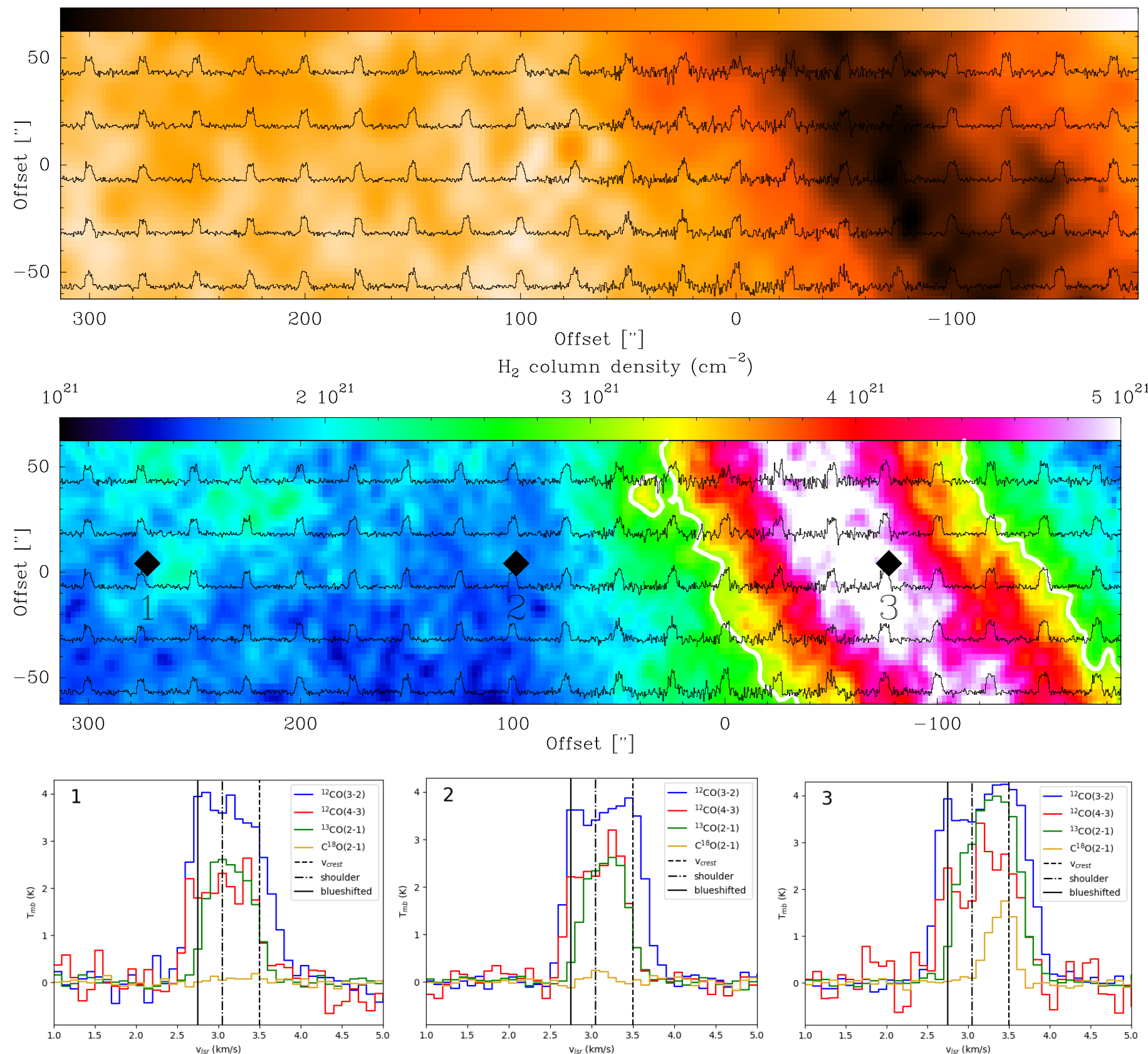

Fig. 4. Top: ${ }^{12} \mathrm{CO}(4-3)$ spectra overlaid on the Herschel dust temperature map. The $(0,0)$ position of the map is $\alpha_{2000}=12^{\mathrm{h}} 28^{\mathrm{m}} 58^{\mathrm{s}}$, $\delta_{2000}=$ $-71^{\circ} 16^{\prime} 55^{\prime \prime}$ Middle: ${ }^{12} \mathrm{CO}(4-3)$ spectra overlaid on the northern Herschel column density map. The white contours indicate Musca filament crest $\left(N>3 \times 10^{21} \mathrm{~cm}^{-2}\right)$. The black squares indicate the locations of the spectra displayed below. Below: zoom into spectra at selected positions in the map. This shows that there is ${ }^{12} \mathrm{CO}(4-3)$ and ${ }^{12} \mathrm{CO}(3-2)$ emission at the velocity of the blueshifted component over the full map.

\subsubsection{Density for the blueshifted component}

From the sections before, we learned that the blueshifted component is an individual feature towards the crest and strand regions, and that the ${ }^{12} \mathrm{CO}(2-1)$ line has contributions from the ambient cloud along the line-of-sight. Here, we estimate the typical density and upper limit for this ambient cloud close to the filament. For that we use the density of the fitted Plummer profile at the outer radius of the filament. With the values from Cox et al. (2016), correcting for a distance of $140 \mathrm{pc}$ for the Musca filament, this gives $n_{\mathrm{H}_{2}}=4.0 \times 10^{2} \mathrm{~cm}^{-3}$ at $r=0.2 \mathrm{pc}$. An approach to estimate the density upper limit is to combine the maximal column density associated with the ambient cloud, which is $N \sim 10^{21} \mathrm{~cm}^{-2}$ (Cox et al. 2016), with the minimal possible size of the ambient cloud. As it is unlikely that the ambient cloud has a smaller size along the line of sight than the filament, this gives a minimal size of $0.4 \mathrm{pc}$. Using a minimal size of $0.4 \mathrm{pc}$ along the line of sight gives an upper limit of $n_{\mathrm{H}_{2}}=8 \times 10^{2} \mathrm{~cm}^{-3}$ for the ambient cloud.

The $\left[\mathrm{C}_{\mathrm{I}}\right]$ emission at the velocity of the blueshifted component in Fig. 3 is weak, $\sim 1-1.5 \mathrm{~K}$, but it is difficult to constrain this because of the noise. This indicates that $\left[\mathrm{C}_{\mathrm{I}}\right]$ can trace the 
Table 1. Linewidth, integrated brightness and velocity for the average ${ }^{12} \mathrm{CO}$ blueshifted component in the northern and southern map after fitting 3 gaussians to the spectrum.

\begin{tabular}{cccc}
\hline \hline line & $F W H M\left(\mathrm{~km} \mathrm{~s}^{-1}\right)$ & $\int T \mathrm{~d} v\left(\mathrm{~K} \mathrm{~km} \mathrm{~s}^{-1}\right)$ & $v_{\text {blue }}\left(\mathrm{km} \mathrm{s}^{-1}\right)$ \\
\hline \multicolumn{5}{c}{ North } \\
\hline${ }^{12} \mathrm{CO}(2-1)$ & 0.35 & 2.0 & 2.7 \\
${ }^{12} \mathrm{CO}(3-2)$ & 0.31 & 0.98 & 2.8 \\
${ }^{12} \mathrm{CO}(4-3)$ & 0.42 & 0.73 & 2.9 \\
\hline \multicolumn{5}{c}{ South } \\
${ }^{12} \mathrm{CO}(2-1)$ & 0.71 & 4.6 & 2.5 \\
${ }^{12} \mathrm{CO}(3-2)$ & 0.35 & 1.7 & 2.5 \\
${ }^{12} \mathrm{CO}(4-3)$ & 0.33 & 0.63 & 2.5 \\
\hline
\end{tabular}

ambient cloud down to at least $n_{\mathrm{H}_{2}} \sim 4 \times 10^{2} \mathrm{~cm}^{-3}$ in a weak FUV-field. More extensive [ $\left.\mathrm{C}_{\mathrm{I}}\right]$ observations will be required to better understand the physical conditions traced by $\left[\mathrm{C}_{\mathrm{I}}\right]$ in the ISM. This is particularly important for comparison with simulations since the physical conditions traced by $\left[\mathrm{C}_{\mathrm{I}}\right]$ remain uncertain in simulations (e.g. Glover et al. 2015; Franeck et al. 2018; Clark et al. 2019).

\section{4. ${ }^{12} \mathrm{CO}(4-3)$ excess emission in the blueshifted velocity component}

The ${ }^{12} \mathrm{CO}(3-2)$ and ${ }^{12} \mathrm{CO}(4-3)$ transitions have relatively high excitation temperatures ( 33 and $55 \mathrm{~K}$ respectively; Müller et al. 2005). This allows us to investigate the presence of warm gas in the Musca cloud. We focus on the blueshifted component for which ${ }^{12} \mathrm{CO}(4-3)$ is observed over the full map. This could be an indication of quite uniform heating by e.g. a FUV field, though there are indications of spatial variations in the data. However, because of the noise in the spatially resolved map, we will not focus on these possible spatial variations in this paper. To further investigate the heating that leads to the ${ }^{12} \mathrm{CO}(4-3)$ emission, we study the ${ }^{12} \mathrm{CO}(4-3) /{ }^{12} \mathrm{CO}(2-1)$ and ${ }^{12} \mathrm{CO}(4-3) /{ }^{12} \mathrm{CO}(3-2)$ brightness temperature ratios. In the previous section, we noted that it is important to take care comparing the integrated brightness of ${ }^{12} \mathrm{CO}(2-1)$ with ${ }^{12} \mathrm{CO}(3-2)$ and ${ }^{12} \mathrm{CO}(4-3)$ in the blueshifted component because of their difference in linewidth. Furthermore, the noise in the ${ }^{12} \mathrm{CO}(3-2)$ and ${ }^{12} \mathrm{CO}(4-3)$ spectra makes it impossible to confidently fit a spectrum for every pixel in the map, even when spatially smoothing the data. Because of this, we will approach this section by focusing on the peak brightness temperatures.

The peak brightness of the blueshifted component occurs near $2.8 \mathrm{~km} \mathrm{~s}^{-1}$ in the northern map and near $2.5 \mathrm{~km} \mathrm{~s}^{-1}$ in the southern map. At these velocities, we determined the brightness temperature ratios for every pixel in the maps. In Fig. 5, the distribution of the ${ }^{12} \mathrm{CO}(4-3) /{ }^{12} \mathrm{CO}(2-1)$ and ${ }^{12} \mathrm{CO}(4-$ $3) /{ }^{12} \mathrm{CO}(3-2)$ peak brightness ratios for the northern map are displayed. We determined the average and median values for the various ratios in both maps which are summarized in Table 2. Overall, there is no large difference between average and median values. For the ${ }^{12} \mathrm{CO}(4-3) /{ }^{12} \mathrm{CO}(2-1)$ and ${ }^{12} \mathrm{CO}(4-3) /{ }^{12} \mathrm{CO}(3-$ 2) ratio in the northern map, we obtain a median value of 0.38 and 0.61 at $2.8 \mathrm{~km} \mathrm{~s}^{-1}$, respectively. In the southern map, the noise of both the ${ }^{12} \mathrm{CO}(4-3)$ and ${ }^{12} \mathrm{CO}(3-2)$ transition is higher as it was observed under poorer weather conditions. The median

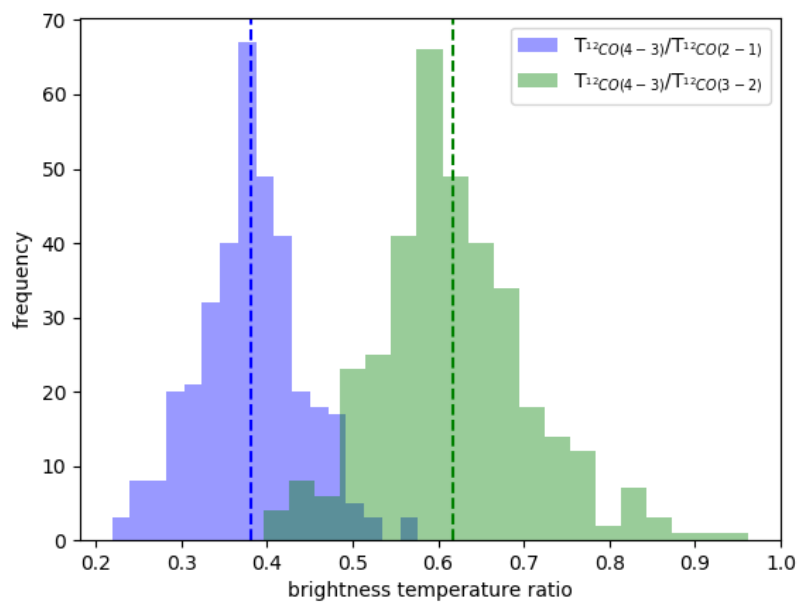

Fig. 5. Histograms of the ${ }^{12} \mathrm{CO}(4-3) /{ }^{12} \mathrm{CO}(2-1)$ (blue) and ${ }^{12} \mathrm{CO}(4-$ $3) /{ }^{12} \mathrm{CO}(3-2)$ (green) main beam brightness ratio for the pixels in the northern map with a ${ }^{12} \mathrm{CO}(4-3) \mathrm{rms}<0.2 \mathrm{~K}$. The dashed blue line indicates the average ${ }^{12} \mathrm{CO}(4-3) /{ }^{12} \mathrm{CO}(2-1)$ ratio for all pixels, and the dashed green line indicates the average ${ }^{12} \mathrm{CO}(4-3) /{ }^{12} \mathrm{CO}(3-2)$ ratio.

Table 2. Mean and median main beam brightness ratios for ${ }^{12} \mathrm{CO}(4$ $3) /{ }^{12} \mathrm{CO}(2-1)$ and ${ }^{12} \mathrm{CO}(4-3) /{ }^{12} \mathrm{CO}(3-2)$ in the blueshifted component for the northern and southern map.

\begin{tabular}{lcccc}
\hline \hline & ${ }^{12} \mathrm{CO}(4-3) /{ }^{12} \mathrm{CO}(2-1)$ & & ${ }^{12} \mathrm{CO}(3-2) /{ }^{12} \mathrm{CO}(2-1)$ \\
\cline { 2 - 3 } \cline { 5 - 5 } Mean & Median & Mean & Median \\
\hline north $\left(2.8 \mathrm{~km} \mathrm{~s}^{-1}\right)$ & $0.38 \pm 0.06$ & 0.38 & $0.62 \pm 0.09$ & 0.61 \\
south $\left(2.5 \mathrm{~km} \mathrm{~s}^{-1}\right)$ & $0.39 \pm 0.13$ & 0.38 & $0.55 \pm 0.19$ & 0.53 \\
\hline
\end{tabular}

${ }^{12} \mathrm{CO}(4-3) /{ }^{12} \mathrm{CO}(2-1)$ ratio is 0.38 at $2.5 \mathrm{~km} \mathrm{~s}^{-1}$ and the respective value for the ${ }^{12} \mathrm{CO}(4-3) /{ }^{12} \mathrm{CO}(3-2)$ ratio is 0.53 .

\subsubsection{Modeling with the PDR Toolbox}

We compared the observed ${ }^{12} \mathrm{CO}(4-3) /{ }^{12} \mathrm{CO}(2-1)$ and ${ }^{12} \mathrm{CO}(4$ $3) /{ }^{12} \mathrm{CO}(3-2)$ brightness temperature ratios in the blueshifted component with predictions using the PDR toolbox in the allowed density range of the ambient cloud in Musca, see Sect. 3.3.2. Figure 6 shows that for a FUV field strength $<1 G_{0}$, the predicted ${ }^{12} \mathrm{CO}(4-3) /{ }^{12} \mathrm{CO}(2-1)$ brightness temperature ratio is smaller than 0.05 , which is more than a factor 5 lower than observed in Musca. The same is found for the ${ }^{12} \mathrm{CO}(4-3) /{ }^{12} \mathrm{CO}(3-2)$ brightness temperature ratio, with predicted ratios of $\sim 0.1$, which is again more than a factor 5 lower than observed. It is thus impossible to obtain the observed ratios for the densities and FUV field strength in the ambient Musca cloud that are allowed by $\left[\mathrm{C}_{\mathrm{II}}\right],\left[\mathrm{C}_{\mathrm{I}}\right],\left[\mathrm{O}_{\mathrm{I}}\right]$, and the calculated upper limit from the nearby census of OB stars. This strongly suggests that the ${ }^{12} \mathrm{CO}(4-3)$ emission in the low-column density blueshifted component cannot be explained as a result of FUV heating.

\subsubsection{Modeling with RADEX}

We further investigate whether the ${ }^{12} \mathrm{CO}(4-3) /{ }^{12} \mathrm{CO}(2-1)$ and ${ }^{12} \mathrm{CO}(4-3) /{ }^{12} \mathrm{CO}(3-2)$ brightness temperature ratios in the blueshifted component can be reproduced with the non-LTE RADEX code (van der Tak et al. 2007). We use a non-LTE 


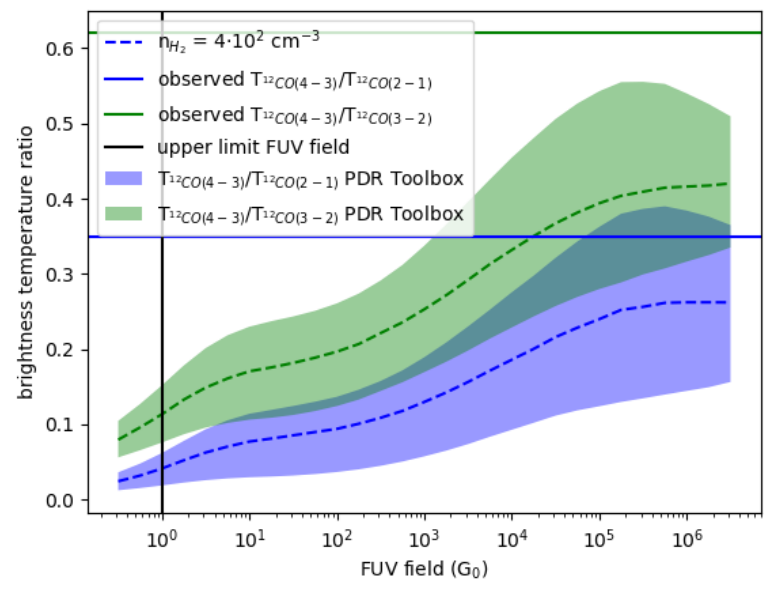

Fig. 6. Observed ${ }^{12} \mathrm{CO}(4-3) /{ }^{12} \mathrm{CO}(2-1)$ and ${ }^{12} \mathrm{CO}(4-3) /{ }^{12} \mathrm{CO}(3-2)$ ratios compared with predictions by the PDR Toolbox. The shaded areas, constructed by logarithmic interpolation between the points in the PDR Toolbox density grid, show the evolution of the ${ }^{12} \mathrm{CO}(4-$ $3) /{ }^{12} \mathrm{CO}(2-1)$ and ${ }^{12} \mathrm{CO}(4-3) /{ }^{12} \mathrm{CO}(3-2)$ brightness temperature ratio as a function of the FUV field in the allowed density range of the Musca cloud. At a typical density $n_{\mathrm{H}_{2}}=4 \times 10^{2} \mathrm{~cm}^{-3}$ of the ambient cloud (dashed line) for the allowed FUV field strength $\left(<1 G_{0}\right)$, the predicted line ratios are only a fraction of the observed ones in the blueshifted component.

approach because the high critical density $\left(n_{\mathrm{H}_{2}}>5 \times 10^{4} \mathrm{~cm}^{-3}\right)$ of ${ }^{12} \mathrm{CO}(3-2)$ and ${ }^{12} \mathrm{CO}(4-3)$ implies that these lines are subthermally excited. For RADEX analysis we use a FWHM of $0.4 \mathrm{~km} \mathrm{~s}^{-1}$, temperatures between 15 and $25 \mathrm{~K}$, and representative densities for the ambient cloud: $n_{\mathrm{H}_{2}}=3 \times 10^{2}, 5 \times 10^{2}$, $7.5 \times 10^{2}$ and $1.5 \times 10^{3} \mathrm{~cm}^{-3}$.

For each RADEX model, we also need an upper limit on the ${ }^{12} \mathrm{CO}$ column density of the blueshifted component. This can be done by calculating with RADEX the ${ }^{13} \mathrm{CO}$ column density from the ${ }^{13} \mathrm{CO}(2-1)$ brightness $(\sim 0.6 \mathrm{~K}$, see Fig. 3) for every density and temperature. In a weak FUV field the $\left[{ }^{12} \mathrm{CO}\right] /\left[{ }^{13} \mathrm{CO}\right]$ abundance ratio is $\leq 60$ (Visser et al. 2009; Röllig \& Ossenkopf 2013), which puts an upper limit on the ${ }^{12} \mathrm{CO}$ column density and opacity. For further analysis, we use RADEX models with a predicted ${ }^{12} \mathrm{CO}(2-1)$ brightness up to $50 \%$ brighter than observed towards Musca to take into account some uncertainties such as possible opacity broadening of ${ }^{12} \mathrm{CO}(2-1)$ compared to ${ }^{12} \mathrm{CO}(4-3)$, a non unity beam filling or different line calibration (uncertainty in $\eta_{\mathrm{mb}}$ ).

Studying the ${ }^{12} \mathrm{CO}(4-3) /{ }^{12} \mathrm{CO}(2-1)$ and ${ }^{12} \mathrm{CO}(4-3) /{ }^{12} \mathrm{CO}(3-$ 2 ) brightness temperature ratios within these limits, we find that the ratio increases with increasing density, column density or temperature, see Fig. 7. However, no model is capable to reproduce more than $40 \%$ of the observed ${ }^{12} \mathrm{CO}(4-3) /{ }^{12} \mathrm{CO}(2-1)$ and ${ }^{12} \mathrm{CO}(4-3) /{ }^{12} \mathrm{CO}(3-2)$ ratios towards the Musca ambient cloud, see Fig. 7 for the ${ }^{12} \mathrm{CO}(4-3) /{ }^{12} \mathrm{CO}(2-1)$ ratio.

Taking higher densities for the ambient cloud than put forward in Sect. 3.3.2, does not offer a solution for bringing calculations in agreement with observations. Though this can increase the predicted ratio for a fixed temperature and ${ }^{12} \mathrm{CO}$ column density, this also strongly increases the line brightness temperature. Consequently, one either has to reduce the temperature or ${ }^{12} \mathrm{CO}$ column density to keep line brightnesses that are not too far off from the observed values. This effort again forces brightness temperature ratios to values found in Fig. 7.

From both approaches, it thus appears impossible to reproduce the observed ${ }^{12} \mathrm{CO}(4-3) /{ }^{12} \mathrm{CO}(2-1)$ and ${ }^{12} \mathrm{CO}(4-3) /$

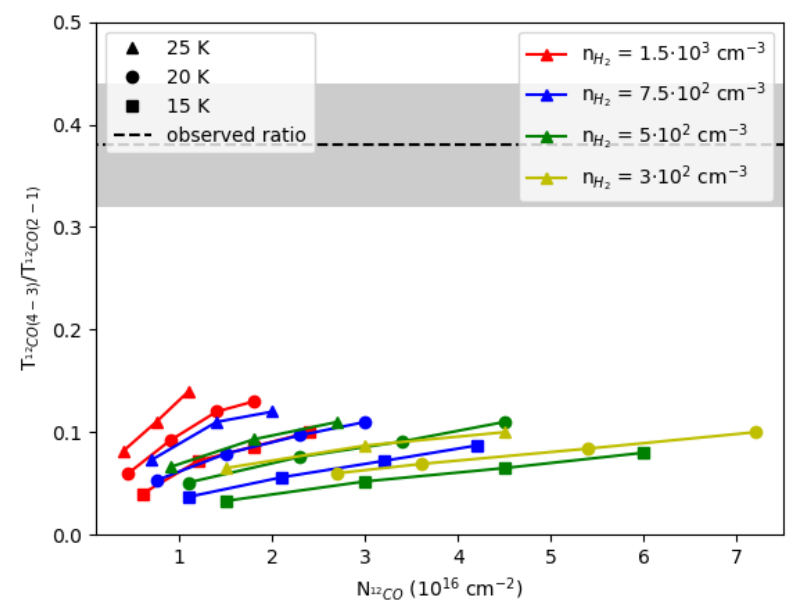

Fig. 7. ${ }^{12} \mathrm{CO}(4-3) /{ }^{12} \mathrm{CO}(2-1)$ brightness temperature ratios predicted with RADEX for the plausible range of physical conditions in the ambient cloud of the Musca filament. The lines connect the RADEX models with identical density and temperature for varying ${ }^{12} \mathrm{CO}$ column densities. The predicted ratios with RADEX show a huge discrepancy with the observed ${ }^{12} \mathrm{CO}(4-3) /{ }^{12} \mathrm{CO}(2-1)$ ratio towards the Musca cloud (indicated by the dashed line). The standard deviation of the observed values from the average is indicated by the grey area. The same is observed for the ${ }^{12} \mathrm{CO}(4-3) /{ }^{12} \mathrm{CO}(3-2)$ brightness temperature ratio.

${ }^{12} \mathrm{CO}(3-2)$ brightness temperature ratios assuming that there is only collisional and radiative heating.

\subsection{A warm and dense gas component}

\subsubsection{The CO line ratio with RADEX}

In order to increase the ${ }^{12} \mathrm{CO}(4-3) /{ }^{12} \mathrm{CO}(2-1)$ and ${ }^{12} \mathrm{CO}(4-$ $3) /{ }^{12} \mathrm{CO}(3-2)$ brightness temperature ratio while keeping the brightness of both lines low enough, one needs to consider a small layer embedded in the diffuse gas that can increase the ${ }^{12} \mathrm{CO}(4-3)$ brightness without significantly increasing the ${ }^{12} \mathrm{CO}(2-1)$ and ${ }^{12} \mathrm{CO}(3-2)$ emission. Based on the ${ }^{12} \mathrm{CO}(4-3)$ excitation conditions, the layer should contain warm gas $(>50 \mathrm{~K})$. In fact, this "layer" can be clumpy, that is, pockets of warm gas that are embedded in more tenuous interclump gas. However, modelling such a scenario is not possible with RADEX and out of the scope of this paper. Our objective here is to show the existence of a warm gas component. We thus investigated with RADEX the impact of a warm gas layer by running models with a FWHM of $0.4 \mathrm{~km} \mathrm{~s}^{-1}$ as an overall average, and temperatures between 10 and $150 \mathrm{~K}$ at different densities. Note that a temperature of $150 \mathrm{~K}$ would result in a thermal FWHM of $0.5 \mathrm{~km} \mathrm{~s}^{-1}$. This is higher than the observed typical FWHM of $0.4 \mathrm{~km} \mathrm{~s}^{-1}$, but we anticipate that there is also uncertainty on the FWHM so that we work with a kinetic temperature upper limit of $150 \mathrm{~K}$. We additionally restrain ourself to models that manage to reproduce the observed ${ }^{12} \mathrm{CO}(4-3)$ brightness temperature in Musca ${ }^{7}$. Figures 8 and 9 show the results for different densities. Both the ${ }^{12} \mathrm{CO}(4-3) /{ }^{12} \mathrm{CO}(2-1)$ and ${ }^{12} \mathrm{CO}(4-3) /{ }^{12} \mathrm{CO}(3-2)$ ratios indicate that a temperature $>50 \mathrm{~K}$ as well as relatively high

7 Using brightness temperatures for modelling is less convenient because of the unknown beam filling factors of $\mathrm{CO}$ emission which are eliminated to first order using line ratios. We nevertheless use the ${ }^{12} \mathrm{CO}(4-3)$ line as an additional - though weak - indicator for the best fitting model. 


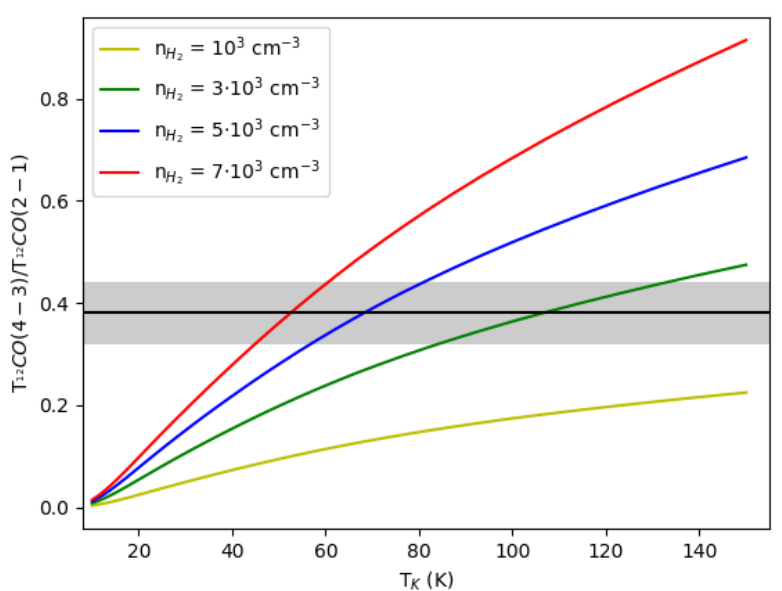

Fig. 8. Predicted brightness temperature ratio of ${ }^{12} \mathrm{CO}(4-3) /{ }^{12} \mathrm{CO}(2-1)$ by RADEX as a function of temperature for different densities. This demonstrates the need for a warm and dense $\mathrm{CO}$ layer to at least reach the observed brightness ratios in the blueshifted component. For each density we plot the ratio for a column density such that the predicted brightness of ${ }^{12} \mathrm{CO}(4-3)$ by RADEX is roughly similar to the observed brightness towards Musca $\left(T_{\mathrm{mb}}=1.5-3 \mathrm{~K}\right)$ at the temperatures that obtain sufficiently high brightness ratios. The lowest three densities $\left(n_{\mathrm{H}_{2}}=10^{3}, 3 \times 10^{3}\right.$ and $\left.5 \times 10^{3} \mathrm{~cm}^{-3}\right)$ use $N_{1_{2} \mathrm{CO}}=1.1 \times 10^{15} \mathrm{~cm}^{-2}$, and $n_{\mathrm{H}_{2}}=7 \times 10^{3} \mathrm{~cm}^{-3}$ uses $N_{{ }^{12} \mathrm{CO}}=9 \times 10^{14} \mathrm{~cm}^{-2}$. The black horizontal line indicates the average observed ratio towards Musca, and the grey area indicates the standard deviation.

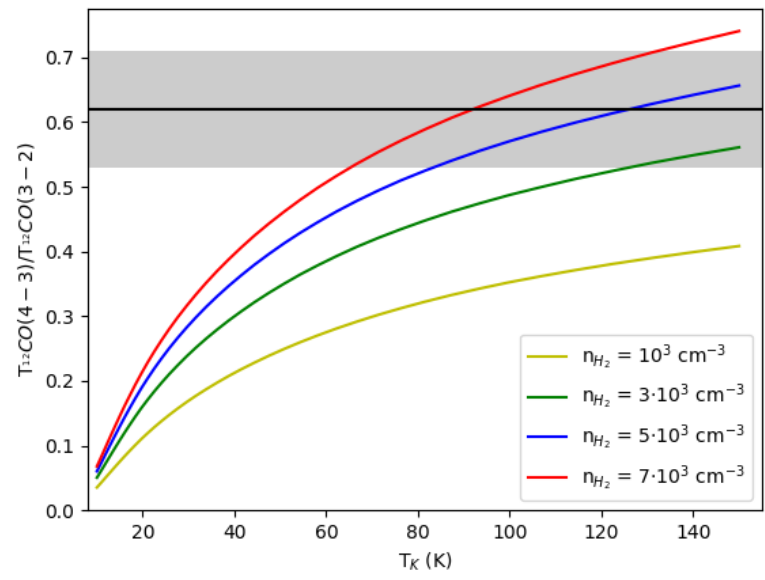

Fig. 9. Predicted brightness temperature ratio of ${ }^{12} \mathrm{CO}(4-3) /{ }^{12} \mathrm{CO}(3-2)$ by RADEX for the same models that are presented in Fig. 8. It confirms the need for high temperatures $(>50 \mathrm{~K})$ and densities $\left(5-7 \times 10^{3} \mathrm{~cm}^{-3}\right)$ to reach the observed excitation of ${ }^{12} \mathrm{CO}(4-3)$. The black horizontal line indicates the average observed ratio towards Musca, and the grey area indicates the standard deviation.

densities are required to reach the observed ratios. The ${ }^{12} \mathrm{CO}(4-$ $3) /{ }^{12} \mathrm{CO}(3-2)$ ratio, in particular, points to high densities and temperature, see Fig. 9, in order to reach the observed ratios.

Though the RADEX analysis demonstrates the need for warm and dense gas to obtain the observed ${ }^{12} \mathrm{CO}(4-3)$ brightness in the blueshifted component, we can not narrow down more precisely the temperature and density range because there are no higher-J CO observations at the moment. This warm and dense gas exactly fits with the predictions for gas heated by low-velocity shocks (Pon et al. 2012; Lesaffre et al. 2013), and so-called slow-type (with regard to their phase velocity) magnetised shocks with $v_{\mathrm{s}}=1-3 \mathrm{~km} \mathrm{~s}^{-1}$ can easily reach temperatures as high as $100 \mathrm{~K}$ (Lehmann \& Wardle 2016). These slow-type
Table 3. Predicted [C I] brightness temperatures (4), calculated with RADEX for various temperatures (1) and densities (2) for the blueshifted velocity component.

\begin{tabular}{cccc}
\hline \hline$T(\mathrm{~K})$ & $n_{\mathrm{H}_{2}}\left(\mathrm{~cm}^{-3}\right)$ & $\mathrm{N}_{\mathrm{CI}}\left(\mathrm{cm}^{-2}\right)$ & $T_{\mathrm{mb}}(\mathrm{K})$ \\
$(1)$ & $(2)$ & $(3)$ & $(4)$ \\
\hline \multicolumn{4}{c}{ Ambient cloud } \\
\hline 20 & $5 \times 10^{2}$ & $10^{16}$ & 1.0 \\
20 & $7.5 \times 10^{2}$ & $10^{16}$ & 1.2 \\
\hline \multicolumn{4}{c}{ Warm gas layer } \\
\hline 60 & $7 \times 10^{3}$ & $9 \times 10^{14}$ & 0.16 \\
90 & $7 \times 10^{3}$ & $9 \times 10^{14}$ & 0.15 \\
\hline
\end{tabular}

Notes. This indicates a [CI] column density (3) of $\sim 10^{16} \mathrm{~cm}^{-2}$ for the ambient cloud, while the contribution from the warm gas layer to the observed $[\mathrm{C} \mathrm{I}]$ emission is negligible.

shock models predict a physical size of the warm gas layer around $10^{15} \mathrm{~cm}$, which fits with the estimated physical sizes for the RADEX models. The estimated size, that is the layer thickness, of the models that manage to reach the observed brightness temperature ratios in Figs. 8 and 9 are $1.3 \times 10^{15} \mathrm{~cm}$ and $2.2 \times 10^{15} \mathrm{~cm}$, respectively at $n_{\mathrm{H}_{2}}=7 \times 10^{3} \mathrm{~cm}^{-3}$ (with $\mathrm{N}^{{ }^{12} \mathrm{CO}}=$ $9 \times 10^{14} \mathrm{~cm}^{-2}$ ) and $5 \times 10^{3} \mathrm{~cm}^{-3}$ (with $\mathrm{N}_{12} \mathrm{CO}=1.1 \times 10^{15} \mathrm{~cm}^{-2}$ ). To calculate the physical size of the RADEX models an abundance of $\left[\mathrm{H}_{2}\right] /\left[{ }^{12} \mathrm{CO}\right]=10^{4}$ was used, which is a typical value for weakly irradiated molecular gas.

Lastly, we note that this warm gas is observed in both maps and thus likely universally present around the Musca filament.

\subsubsection{Cl column density in the blueshifted component}

We noted in Fig. 3 the presence of [C $\mathrm{I}]$ emission in the blueshifted component with a brightness temperature of the order of $1-1.5 \mathrm{~K}$. From this weak [ $\left.\mathrm{C}_{\mathrm{I}}\right]$ emission we here estimate the $\left[\mathrm{C}_{\mathrm{I}}\right]$ column density. We use a $F W H M=0.4 \mathrm{~km} \mathrm{~s}^{-1}$, a temperature of $20 \mathrm{~K}$, and typical densities $n_{\mathrm{H}_{2}}=5-7.5 \times 10^{2} \mathrm{~cm}^{-3}$ for the ambient Musca cloud with RADEX. This points to $\mathrm{N}_{\mathrm{CI}} \sim 10^{16} \mathrm{~cm}^{-2}$, see Table 3. Comparing this with the same models for ${ }^{12} \mathrm{CO}$ emission from the ambient cloud in Sect. 3.4, we find that at least $20 \%$ and possibly up to $50 \%$ of carbon is still found in its atomic form.

On the other hand, it is observed from Table 3 that the warm and dense gas layer, necessary to explain the bright ${ }^{12} \mathrm{CO}(4-3)$ emission, provides a negligible contribution to the [C I] emission.

We thus emphasise again that the blueshifted component has two contributions:

- the ambient cloud which gives rise to $\left[\mathrm{C}_{\mathrm{I}}\right]$ and low-J CO emission;

- a warm gas layer (or pockets of warm gas) with little [C $\mathrm{I}]$ emission that is responsible for the bright $\mathrm{CO}(4-3)$ line.

\subsection{Shock models}

For an in-depth comparison with shock models, it would be preferable to have additional observations of mid-to high-J $\left(J_{\text {up }}>4\right)$ CO lines (e.g. Pon et al. 2012; Lehmann \& Wardle 2016) since ${ }^{12} \mathrm{CO}(2-1)$, and possibly some ${ }^{12} \mathrm{CO}(3-2)$, emission in the blueshifted component also comes from non-shocked 


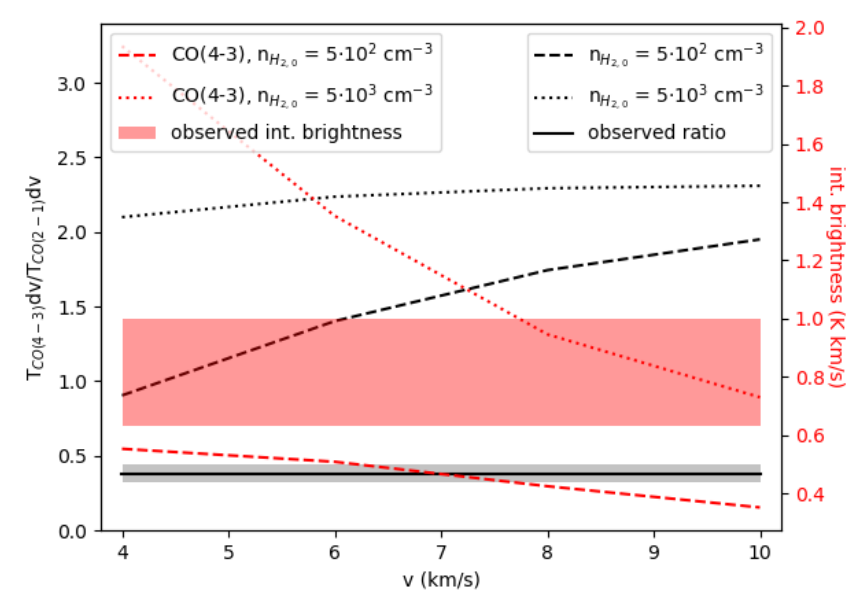

Fig. 10. ${ }^{12} \mathrm{CO}(4-3) /{ }^{12} \mathrm{CO}(2-1)$ integrated brightness ratio (black) for J-type shock models from the Paris-Durham code. The ratio at a shock velocity of $4 \mathrm{~km} \mathrm{~s}^{-1}$ is higher than observed in Musca. This is expected because the ratio decreases towards lower velocities and the ${ }^{12} \mathrm{CO}(2$ 1) emission in Musca also comes from non shocked gas, such that the observed ratio is a lower limit for the shock models. In red, we show the integrated brightness of ${ }^{12} \mathrm{CO}(4-3)$ for the same shock models, together with the ${ }^{12} \mathrm{CO}(4-3)$ integrated brightness interval of the blueshifted component in Musca. The observed values in Musca show a relatively good agreement with the predicted ${ }^{12} \mathrm{CO}(4-3)$ integrated brightness for a pre-shock density of $n_{\mathrm{H}_{2,0}} \sim 5 \times 10^{2} \mathrm{~cm}^{-3}$.

gas (as it is found in synthetic observations of simulations; see Fig. 13).

Because observational studies so far focussed on the higher-J CO lines observed with Herschel (Pon et al. 2014; Larson et al. 2015), it is not well investigated to which extent mid-J CO lines, in particular the ${ }^{12} \mathrm{CO}(4-3)$ transition, contribute as a cooling line for shocks. We thus compared our observations to results of the Paris-Durham shock code (Flower \& Pineau des Forêts 2003; Lesaffre et al. 2013; Godard et al. 2019) that are available for both non-irradiated C- and J-type shocks.

Looking into computed model grids, we find that nonirradiated C-type shocks with a pre-shock density $n_{\mathrm{H}_{2,0}} \sim$ $5 \times 10^{2} \mathrm{~cm}^{-3}$ do not manage to reproduce the observations. Both the integrated intensity of ${ }^{12} \mathrm{CO}(4-3)$ and the predicted ratios are below the observed values in Musca. However, J-type shocks, which are good first order models for slow-type shocks (Lehmann \& Wardle 2016), with a pre-shock density $n_{\mathrm{H}_{2,0}} \sim$ $5 \times 10^{2} \mathrm{~cm}^{-3}$ fit with the ${ }^{12} \mathrm{CO}$ observations, see Fig. 10 . The predictions by these models for the $\left[\mathrm{C}_{\mathrm{II}}\right],\left[\mathrm{O}_{\mathrm{I}}\right]$, and $\left[\mathrm{C}_{\mathrm{I}}\right]$ brightnesses are also in agreement with the observations of Musca (see Appendix A). J-type shocks as a proxy for slow-type low-velocity shocks are justified since the dynamics of these slow-type shocks are driven by the gas pressure. Note that we used published shock models from the Paris-Durham code for this first comparison which have shock velocities of $4 \mathrm{~km} \mathrm{~s}^{-1}$ and higher, while in Musca the shock velocity can be lower. A comparison with shock models at lower velocities is work in progress, and will be addressed in a forthcoming paper.

The nondimensional parameter $b=(\mathrm{B} / 1 \mu \mathrm{G}) / \sqrt{n \mathrm{~cm}^{-3}}=0.1$ in the shock models, with $\mathrm{B}$ the component of the magnetic field that is perpendicular to the direction of shock propogation, is used to model J-type shocks, and $b=1$ is used to model C-type shocks (Lesaffre et al. 2013).

To perform a first estimate of the shock velocity, we have to take into account that there is an orientation angle for the shock propagation with respect to the plane of the sky (POS). We will assume shock propagation along the magnetic field. In this scenario, one can estimate the true shock velocity when the angle between the magnetic field and the POS is known, using

$v_{\text {shock }}=v_{\text {los }} / \sin (\gamma)$,

where $\gamma$ is the angle between the magnetic field and the POS, and $v_{\text {los }}$ is the observed shock velocity along the line of sight for which we take $0.4 \mathrm{~km} \mathrm{~s}^{-1}$. In Planck Collaboration Int. XXXIII (2016), a value around $25^{\circ}$ is put forward for the angle between the magnetic field and the POS. This results in a shock velocity around $0.9 \mathrm{~km} \mathrm{~s}^{-1}$. This fits nicely with the proposed presence of slow-type low-velocity shocks, which need to have velocities between the sound speed $\left(c_{\mathrm{s}}\right)$ and $v_{\mathrm{A}} \cos (\theta)$. With $v_{\mathrm{A}}$ the alfvén speed and $\theta$ the angle between the magnetic field and the propagation direction of the shock (Lehmann \& Wardle 2016). The expected alfvén speed towards the ambient cloud in Musca is of the order of $2.2 \mathrm{~km} \mathrm{~s}^{-1}$, assuming a $33 \mu \mathrm{G}$ magnetic field strength at $n_{\mathrm{H}_{2}} \sim 4 \times 10^{2} \mathrm{~cm}^{-3}$ (Crutcher 2012). We thus find that the estimated shock velocity fits with the conditions that allow a slow-type shock. An angle $<10^{\circ}$ between the magnetic field and the plane of the sky is required for a shock velocity $>2.2 \mathrm{~km} \mathrm{~s}^{-1}$.

Summarising, the $\mathrm{CO}$ brightness temperature ratios that we observe in Musca are consistent with the predictions from lowvelocity shock models (e.g. Pon et al. 2012; Lehmann \& Wardle 2016). Low- to mid-J CO lines may thus indeed be used to detect low-velocity shocks but more observational studies are required to better understand the quantitative contribution from shock excitation.

Since $\mathrm{SiO}$ transitions can be used as a tracer of certain shocks (e.g. Schilke et al. 1997; Gusdorf et al. 2008a,b), it is worth noting the $\mathrm{SiO}(5-4)$ line was not detected towards the Musca filament. With RADEX this allows to put a $3 \sigma$ upper limit on the $\mathrm{SiO}$ column density of $\sim 10^{14} \mathrm{~cm}^{-2}$ at the filament crest, assuming $n_{\mathrm{H}_{2}}=10^{4} \mathrm{~cm}^{-3}$. This implies that less than $0.06 \%$ of $\mathrm{Si}$ is in the form of $\mathrm{SiO}$ at the Musca filament crest (see Appendix $\mathrm{C}$ for more information). Taking that all the gas-phase silicon is found in the form of $\mathrm{SiO}$ at the crest (Louvet et al. 2016), suggests that all $\mathrm{Si}$ is locked in the grains of the Musca cloud at an early stage of evolution. To explain the presence of $\mathrm{SiO}$ in the gas phase, as is found in regions like W43 (Louvet et al. 2016), implies that there is the need for an important dynamical or radiative history in such clouds to release $\mathrm{Si}$ from the grains in the gas phase.

\section{A filament accretion shock signature in simulations}

From the analysis of the observations, we found the presence a of warm gas layer around the dense gas (filament crest and strands) in the Musca cloud. These findings are here compared with synthetic observations from a converging flow simulation of filament formation (Clarke et al. 2018). We emphasize that this is a qualitative comparison to investigate the role of lowvelocity shocks in filament formation, because the simulation was set up for simulating slightly denser features such as the filaments in Taurus. Nevertheless, we find in the simulations some generic features related to filament accretion that are observed in Musca. In these simulations a radially convergent flow forms dense filaments with $n_{\mathrm{H}_{2}} \gtrsim 10^{4} \mathrm{~cm}^{-3}$, see Figs. 11 and 13. The filamentary structure, that continuously accretes mass from this converging flow, has a size of 3-4 pc. Turbulence in the flow leads to structure formation in the converging flow, causing inhomogeneities in the accretion and subsequent substructure in the 

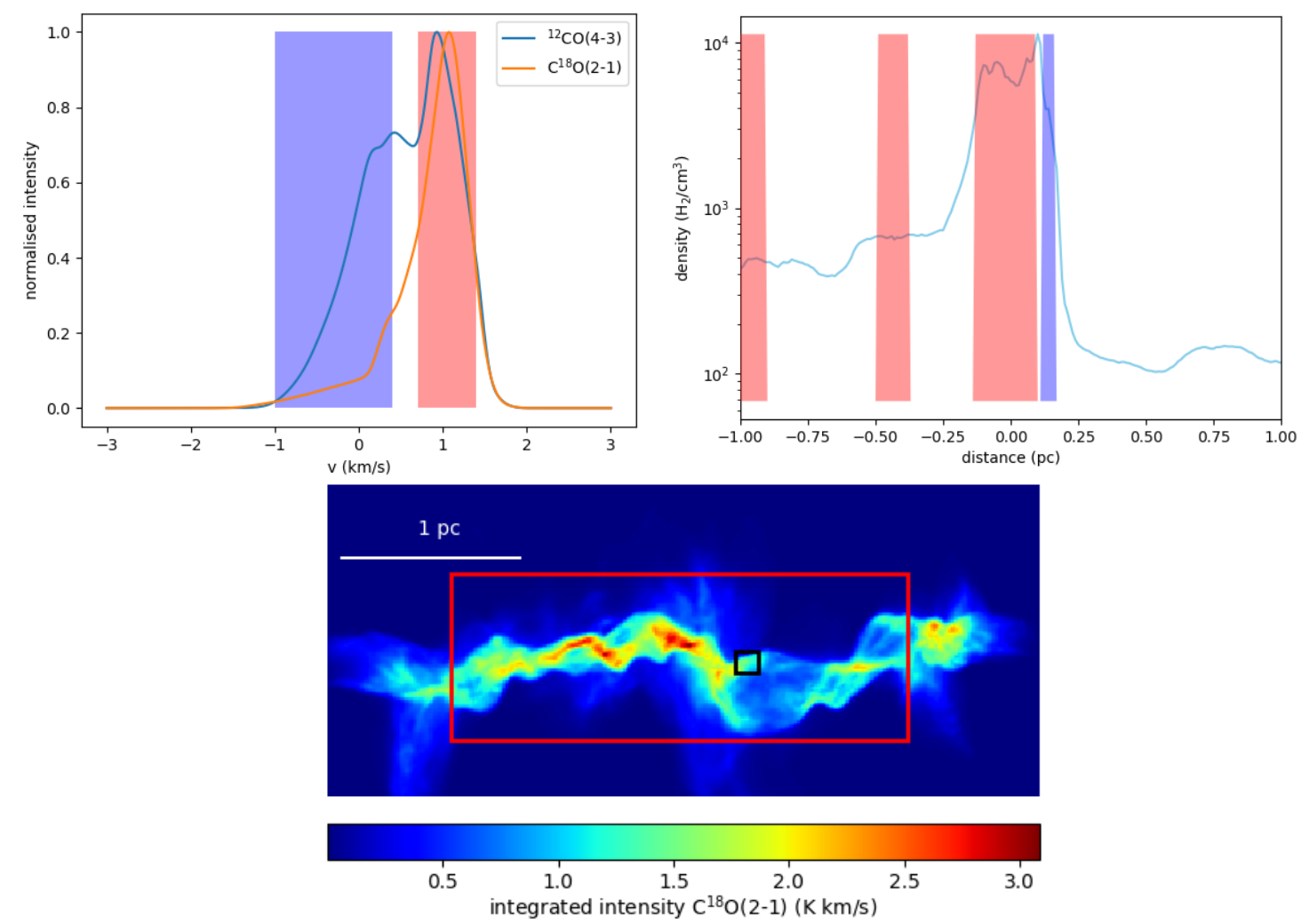

Fig. 11. Bottom: $\mathrm{C}^{18} \mathrm{O}(2-1)$ integrated intensity map of the simulation (Clarke et al. 2018), with the black square indicating the region that is studied in this figure. The red box indicates the region displayed in Fig. 12. Left: ${ }^{12} \mathrm{CO}(4-3)$ and $\mathrm{C}^{18} \mathrm{O}(2-1)$ spectra extracted from the square which show a blueshifted ${ }^{12} \mathrm{CO}(4-3)$ component (blue) in addition to the component related to the bulk emission of the filament crest (red). The two velocity intervals used for the plot on the right are indicated in blue and red. Right: physical location along the line of sight of the gas in the velocity intervals, showing that the larger blueshifted velocity interval covers a small region compared to the filament and that it is located at the border of this filament where the density strongly increases because of a filament accretion shock.

filament. The simulation includes hydrodynamics, self-gravity, and heating and cooling coupled with non-equilibrium chemistry. From this self-consistent $\mathrm{CO}$ formation in the simulations, synthetic observations are produced.

The filament edge is defined by filament accretion shocks, see for example Fig. 11, which is a low-velocity shock due to mass accretion on an interstellar filament from a converging flow in a molecular cloud. For more information on these simulations and synthetic observations, we refer to Clarke et al. (2017, 2018).

No magnetic field is included in the simulations, which could have an impact on the shock properties (e.g. Draine et al. 1983; Lesaffre et al. 2013). For a shock propagating perpendicular to the magnetic field, this makes the shock wider and decreases the peak temperature. However, slow-type shocks, which can fit the observed emission, tend to resemble non-magnetised shocks because the dynamics is driven by gas pressure (Lehmann \& Wardle 2016). In the simulations the warm post-shock layer is not resolved, as post-shock cooling of a low-velocity J-type shock occurs over small distances (e.g. Lesaffre et al. 2013; Whitworth \& Jaffa 2018), implying that the gas heated by the shocks is smeared out. Consequently, the temperature of the heated gas is underestimated. Nonetheless, it is generally observed in the simulations that the 3D dense filamentary structure is confined by a gas layer that shows an increase in temperature as a result of filament accretion shocks. Figure 12 shows a temperature map, cut out of the simulation, that illustrates this temperature increase at the border of the filament, followed by a temperature drop into the filament.

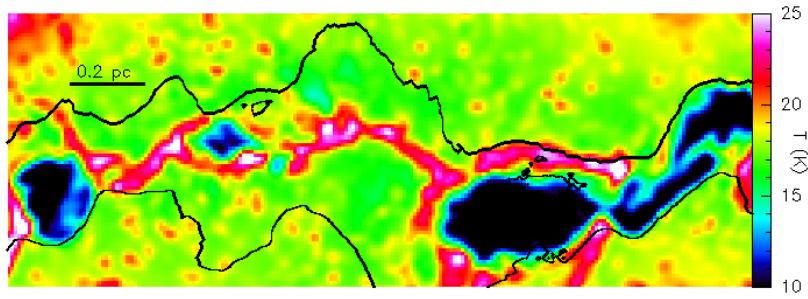

Fig. 12. Smoothed $(\sigma=1.3 \mathrm{pix})$ cut at $z=0.05 \mathrm{pc}$ through the temperature cube of the simulation, with the black contours $\left(N=5 \times 10^{21} \mathrm{~cm}^{-2}\right)$ roughly indicating the high column density filament. Note the local increase in temperature at the sub-filament border due to the accretion shocks and the temperature drop in the sub-filaments after the shock compared to the inflowing mass reservoir. Because of smoothing, the temperature peaks of the shocked gas in the map have decreased. The area of the simulation that is covered by this figure is indicated in Fig. 11.

The good correspondence with synthetic spectra derived from the simulation is remarkable, see Fig. 11. We observe the bulk emission of the filament with the $\mathrm{C}^{18} \mathrm{O}(2-1)$ line and, more importantly, a bright blueshifted component in ${ }^{12} \mathrm{CO}(4-3)$ with no $\mathrm{C}^{18} \mathrm{O}$ emission that connects the velocity of the inflowing cloud and the dense gas. This is similar to what is found in the Musca filament. In the simulations, this ${ }^{12} \mathrm{CO}(4-3)$ component corresponds to a strong velocity transition and density increase from a filament accretion shock of the inflowing mass reservoir, see Fig. 11. This accretion shock is responsible for the 

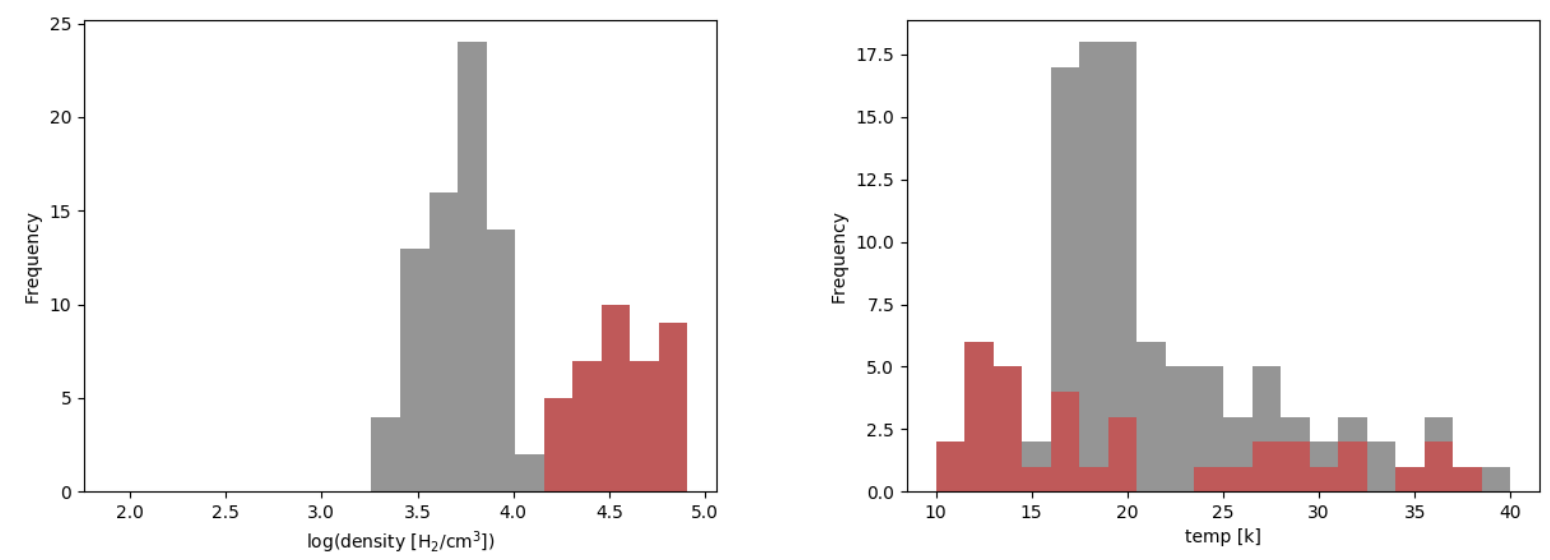

Fig. 13. Left: density distribution of the gas with a sufficient $\mathrm{CO}$ abundance to be observable in a ${ }^{12} \mathrm{CO}(4-3)$ excess component. $\mathrm{CO}$ is considered detectable if more than half of the carbon is locked in CO. Note that the density distribution is relatively bimodal, with the densest already shocked gas highlighted in red. Right: temperature distribution of all the $\mathrm{CO}$ gas in grey, with in red the temperature of the high density gas highlighted on the left. We find for the dense gas that almost all gas at $T=15-25 \mathrm{~K}$ has disappeared, leaving only the cold $(<15 \mathrm{~K})$ and warm gas $(>25 \mathrm{~K})$. This demonstrates that post-shock gas is either warm or cooled behind the shocked gas layer compared to the temperature of the inflowing mass reservoir.

observed blueshifted ${ }^{12} \mathrm{CO}(4-3)$ component. It can be wondered how the slightly lower temperatures in the simulation compare to the estimated values for Musca give rise to the same signature. But as mentioned before, the simulations were designed for somewhat higher densities than Musca, which increases the ${ }^{12} \mathrm{CO}(4-3)$ emission and thus compensates for the slightly lower temperatures.

Since the dense filaments in the simulation are confined by an accretion shock, it is not surprising that the ${ }^{12} \mathrm{CO}(4-3)$ excess is found at both observed locations in Musca as it likely confines the entire filament. Figure 12 displays that behind the heated gas layer by the filament accretion shock there is also a sharp decrease in temperature from $\sim 15$ to $\sim 10 \mathrm{~K}$. This creation of cold and dense gas through a filament accretion shock can also be observed in the temperature histograms of the simulations where the dense gas, created by an accretion shock, is either warmer $(>25 \mathrm{~K})$ or significantly colder $(\sim 10 \mathrm{~K})$ than the inflowing cloud ( $\sim 15-17 \mathrm{~K})$, see Fig. 13. This demonstrates that accretion shocks from a continuously inflowing cloud, as observed in Musca, play an essential role in the cooling of the dense star forming ISM. This cooling is related to the increased shielding of the dense gas and the increased cooling rate by $\mathrm{CO}$ due to the higher density, since $\mathrm{CO}$ is an important coolant of molecular clouds (e.g. Goldsmith \& Langer 1978; Whitworth \& Jaffa 2018).

\section{Filament formation due to the dissipation of MHD flows}

We have put forward that the ${ }^{12} \mathrm{CO}(4-3) /{ }^{12} \mathrm{CO}(2-1)$ and ${ }^{12} \mathrm{CO}(4$ $3) /{ }^{12} \mathrm{CO}(3-2)$ excess in the blueshifted component can be explained by the presence of warm, dense gas that has properties predicted by low-velocity shock models. In particular the observed emission can fit with slow-type shocks, while simulations show that these slow-type shocks typically occur at relatively low velocities of $v_{\mathrm{s}}<3 \mathrm{~km} \mathrm{~s}^{-1}$ found in Musca (Lehmann et al. 2016; Park \& Ryu 2019). Furthermore, as the flow near the filament is expected to be well aligned with the magnetic field (Cox et al. 2016), the shock velocity upper limit for slow-type shocks $\left(v_{\mathrm{A}} \cos \theta\right)$ reaches a maximum. This increases the possibility for slow-type shocks to occur. A small, but significant, change in orientation of the magnetic field takes place from the mass reservoir to the filament in Musca (Planck Collaboration Int. XXXIII 2016) which could be the result of low-velocity shocks (Fogerty et al. 2017). Lastly, the observed warm and dense gas layer connects the velocity of the ambient cloud and dense gas in Musca, which indicates that the accretion shock dissipates the kinetic energy of the supersonic converging flow and increases the cooling rate to form a (trans-)sonic dense and cold filament.

If the Musca filament is indeed confined by the accretion shocks, we can do a first check whether the current results give a reasonable estimated mass accretion rate. The mass accretion rate through this low-velocity shock is estimated in Appendix D, giving a mass accretion rate $\gtrsim 20 M_{\odot} \mathrm{pc}^{-1} \mathrm{Myr}^{-1}$. This is similar to the typical values reported for mass inflow towards filaments that will form low-mass stars (e.g. Palmeirim et al. 2013), and would imply that Musca can accrete its current mass in $1 \mathrm{Myr}$.

\section{Summary}

In this paper we present observations of $\left[\mathrm{C}_{\mathrm{II}}\right]$ and $\left[\mathrm{O}_{\mathrm{I}}\right]$ with SOFIA, and observations of ${ }^{12} \mathrm{CO}(4-3),{ }^{12} \mathrm{CO}(3-2)$ and $\left[\mathrm{C}_{\mathrm{I}}\right]$ with APEX towards the Musca filament. Studying these important cooling lines, we find that Musca has an extremely weak FUV field $\left(<1 \mathrm{G}_{0}\right)$, confirmed by determining the FUV field with a census of ionising stars. We further estimated a density $\mathrm{n}_{\mathrm{H}_{2}} \sim 5 \times 10^{2} \mathrm{~cm}^{-3}$ for the ambient gas near the Musca filament, and found that 20 to $50 \%$ of carbon in the ambient cloud is still in atomic form. In the interface region between the ambient cloud and dense gas in the crest and strands, blueshifted excess emission was found with the ${ }^{12} \mathrm{CO}(4-3),(3-2)$, and (2-1) lines.

A non-LTE radiative transfer study with RADEX points to the existence of a small warm $(\gtrsim 50 \mathrm{~K})$ gas layer at relatively high density $\left(n_{\mathrm{H}_{2}} \geq 3 \times 10^{3} \mathrm{~cm}^{-3}\right)$. This "layer" can actually be clumpy with pockets of warm gas embedded in a cooler interclump phase. This excess emission fits with predictions for non-irradiated slow-type low-velocity shock models with a preshock density $n_{\mathrm{H}_{2,0}} \sim 5 \times 10^{2} \mathrm{~cm}^{-3}$. The estimated shock velocity also fits with the shock velocity interval for slow-type shocks.

Comparing the location of the shock emission in the spectra with synthetic observations in simulations of filament formation, we obtain a scenario where the dense Musca filament is formed 
by low-velocity filament accretion shocks in a colliding flow. These low-velocity MHD filament accretion shocks dissipate the supersonic kinetic energy of the colliding flow, creating a (trans-)sonic dense filament. Because of increased shielding and efficient $\mathrm{CO}$ cooling in the dense post-shock gas, these accretion shocks play an important role in cooling the dense filamentary ISM that can form stars in the near future.

Acknowledgements. We thank the anonymous referee for a critical review of the paper and providing useful comments that improved the quality and clarity of this paper. This work was supported by the Agence National de Recherche (ANR/France) and the Deutsche Forschungsgemeinschaft (DFG/Germany) through the project GENESIS (ANR-16-CE92-0035-01/DFG1591/2-1). L.B. also acknowledges support from the Région Nouvelle-Aquitaine. N.S. acknowledges support from the BMBF, Projekt Number 50OR1714 (MOBS-MOdellierung von Beobachtungsdaten SOFIA). We also appreciate support by the German Deutsche Forschungsgemeinschaft, DFG project number SFB 956. We thank M. Röllig, V. Ossenkopf-Okada and J. Stutzki for fruitful discussions on PDR and shock excitation, and self-absorption effects. We also thank F. Wyrowski for providing information to correct the frequency shift in the APEX FLASH observations. This work is based on observations made with the NASA/DLR Stratospheric Observatory for Infrared Astronomy (SOFIA). SOFIA is jointly operated by the Universities Space Research Association, Inc. (DSI) under DLR contract 50 OK 0901 to the University of Stuttgart. This publication is based on data acquired with the Atacama Pathfinder Experiment (APEX) under programme IDs 0100.C-0825(A), 0101.F-9511(A) and 0102.F-9503(A). APEX is a collaboration between the Max-Planck-Institut fur Radioastronomie, the European Southern Observatory, and the Onsala Space Observatory. This research has made use of the SIMBAD database, operated at CDS, Strasbourg, France.

\section{References}

Alves de Oliveira, C., Schneider, N., Merín, B., et al. 2014, A\&A, 568, A98 André, P., Men'shchikov, A., Bontemps, S., et al. 2010, A\&A, 518, L102

André, P., Di Francesco, J., Ward-Thompson, D., et al. 2014, Protostars and Planets VI (Tucson, AZ: University of Arizona Press), 27

Arzoumanian, D., André, P., Didelon, P., et al. 2011, A\&A, 529, L6

Bisbas, T. G., Tanaka, K. E. I., Tan, J. C., Wu, B., \& Nakamura, F. 2017, ApJ, 850,23

Bron, E., Le Bourlot, J., \& Le Petit F. 2014, A\&A, 569, A100

Cartledge, S. I. B., Lauroesch, J. T., Meyer, D. M., \& Sofia, U. J. 2004, ApJ, 613, 1037

Clark, P. C., Glover, S. C. O., Ragan, S. E., \& Duarte-Cabral, A. 2019, MNRAS, 486,4622

Clarke, S. D., Whitworth, A. P., Duarte-Cabral, A., \& Hubber, D. A. 2017, MNRAS, 468, 2489

Clarke, S. D., Whitworth, A. P., Spowage, R. L., et al. 2018, MNRAS, 479, 1722

Cox, N. L. J., Arzoumanian, D., André, P., et al. 2016, A\&A, 590, A110

Crutcher, R. M. 2012, ARA\&A, 50, 29

Csengeri, T., Leurini, S., Wyrowski, F., et al. 2016, A\&A, 586, A149

Draine, B. T., Roberge, W. G., \& Dalgarno, A. 1983, ApJ, 264, 485

Duarte-Cabral, A., Bontemps, S., Motte, F., et al. 2014, A\&A, 570, A1

Federrath, C. 2016, MNRAS, 457, 375

Fitzpatrick, E. L., \& Massa, D. 2005, AJ, 129, 1642

Flower, D. R., \& Pineau des Forêts, G. 2003, MNRAS, 343, 390

Flower, D. R., \& Pineau Des Forêts, G. 2010, MNRAS, 406, 1745

Fogerty, E., Carroll-Nellenback, J., Frank, A., Heitsch, F., \& Pon, A. 2017, MNRAS, 470, 2938

Franco, G. A. P. 1991, A\&A, 251, 581

Franeck, A., Walch, S., Seifried, D., et al. 2018, MNRAS, 481, 4277

Glover, S. C. O., \& Smith, R. J. 2016, MNRAS, 462, 3011

Glover, S. C. O., Clark, P. C., Micic, M., \& Molina, F. 2015, MNRAS, 448, 1607

Godard, B., Pineau des Forêts, G., Lesaffre, P., et al. 2019, A\&A, 622, A100

Goldsmith, P. F. 2001, ApJ, 557, 736

Goldsmith, P. F., \& Langer, W. D. 1978, ApJ, 222, 881

Goldsmith, P. F., \& Langer, W. D. 1999, ApJ, 517, 209

Goldsmith, P. F., Heyer, M., Narayanan, G., et al. 2008, ApJ, 680, 428

Gómez, G. C., \& Vázquez-Semadeni, E. 2014, ApJ, 791, 124

Grevesse, N., \& Sauval, A. J. 1998, Space Sci. Rev., 85, 161

Guevara, C., Stutzki, J., Ossenkopf-Okada, V., et al. 2020, A\&A, 636, A16

Güsten, R., Nyman, L. A., Schilke, P., et al. 2006, A\&A, 454, L13

Gusdorf, A., Cabrit, S., Flower, D. R., \& Pineau Des Forêts, G. 2008a, A\&A, 482, 809
Gusdorf, A., Pineau Des Forêts, G., Cabrit, S., \& Flower, D. R. 2008b, A\&A, 490, 695

Gusdorf, A., Anderl, S., Lefloch, B., et al. 2017, A\&A, 602, A8

Habing, H. J. 1968, Bull. Astron. Inst. Netherlands, 19, 421

Hacar, A., Kainulainen, J., Tafalla, M., Beuther, H., \& Alves, J. 2016, A\&A, 587, A97

Hennebelle, P. 2013, A\&A, 556, A153

Henning, T., Linz, H., Krause, O., et al. 2010, A\&A, 518, L95

Heyer, M., Goldsmith, P. F., Yıldı, U. A., et al. 2016, MNRAS, 461, 3918

Hincelin, U., Wakelam, V., Hersant, F., et al. 2011, A\&A, 530, A61

Hollenbach, D., \& McKee, C. F. 1989, ApJ, 342, 306

Hollenbach, D. J., \& Tielens, A. G. G. M. 1997, ARA\&A, 35, 179

Inoue, T., Hennebelle, P., Fukui, Y., et al. 2018, PASJ, 70, S53

Ivlev, A. V., Silsbee, K., Sipilä, O., \& Caselli, P. 2019, ApJ, 884, 176

Jappsen, A. K., Klessen, R. S., Larson, R. B., Li, Y., \& Mac Low, M. M. 2005, A\&A, 435, 611

Jenkins, E. B. 2009, ApJ, 700, 1299

Jiménez-Serra, I., Caselli, P., Tan, J. C., et al. 2010, MNRAS, 406, 187

Kainulainen, J., Hacar, A., Alves, J., et al. 2016, A\&A, 586, A27

Kaufman, M. J., Wolfire, M. G., \& Hollenbach, D. J. 2006, ApJ, 644, 283

Klein, T., Ciechanowicz, M., Leinz, C., et al. 2014, IEEE Trans.Terahertz Sci. Technol., 4, 588

Könyves, V., André, P., Men'shchikov, A., et al. 2015, A\&A, 584, A91

Kurucz, R. L. 1979, ApJS, 40, 1

Larson, R. L., Evans Neal J., I., Green, J. D., \& Yang, Y.-L. 2015, ApJ, 806, 70 Le Bourlot, J., Le Petit, F., Pinto, C., Roueff, E., \& Roy, F. 2012, A\&A, 541, A76 Le Petit, F., Nehmé, C., Le Bourlot, J., \& Roueff, E. 2006, ApJS, 164, 506

Lehmann, A., \& Wardle, M. 2016, MNRAS, 455, 2066

Lehmann, A., Federrath, C., \& Wardle, M. 2016, MNRAS, 463, 1026

Lesaffre, P., Pineau des Forêts, G., Godard, B., et al. 2013, A\&A, 550, A106

Liszt, H. S. 2017, ApJ, 835, 138

Liszt, H. S., \& Ziurys, L. M. 2012, ApJ, 747, 55

Liszt, H. S., Lucas, R., \& Pety, J. 2006, A\&A, 448, 253

Louvet, F., Motte, F., Gusdorf, A., et al. 2016, A\&A, 595, A122

Malinen, J., Montier, L., Montillaud, J., et al. 2016, MNRAS, 460, 1934

Marsh, K. A., Kirk, J. M., André, P., et al. 2016, MNRAS, 459, 342

Meyer, D. M., Jura, M., \& Cardelli, J. A. 1998, ApJ, 493, 222

Molinari, S., Swinyard, B., Bally, J., et al. 2010, A\&A, 518, L100

Müller, H. S. P., Schlöder, F., Stutzki, J., \& Winnewisser, G. 2005, J. Mol. Struct., 742,215

Nagai, T., Inutsuka, S.-i., \& Miyama, S. M. 1998, ApJ, 506, 306

Nguyen-Lu'o'ng, Q., Motte, F., Carlhoff, P., et al. 2013, ApJ, 775, 88

Padoan, P., Juvela, M., Goodman, A. A., \& Nordlund, A. 2001, ApJ, 553, 227

Palmeirim, P., André, P., Kirk, J., et al. 2013, A\&A, 550, A38

Park, J., \& Ryu, D. 2019, ApJ, 875, 2

Pecaut, M. J., \& Mamajek, E. E. 2013, ApJS, 208, 9

Planck Collaboration Int., XXXIII. 2016, A\&A, 586, A136

Polychroni, D., Schisano, E., Elia, D., et al. 2013, ApJ, 777, L33

Pon, A., Johnstone, D., \& Kaufman, M. J. 2012, ApJ, 748, 25

Pon, A., Johnstone, D., Kaufman, M. J., Caselli, P., \& Plume, R. 2014, MNRAS, 445, 1508

Pound, M. W., \& Wolfire, M. G. 2008, ASP Conf. Ser., 394, 654

Rayner, T. S. M., Griffin, M. J., Schneider, N., et al. 2017, A\&A, 607, A22

Risacher, C., Güsten, R., Stutzki, J., et al. 2018, J. Astron. Instrum., 7, 1840014

Röllig, M., \& Ossenkopf, V. 2013, A\&A, 550, A56

Röllig, M., Abel, N. P., Bell, T., et al. 2007, A\&A, 467, 187

Schilke, P., Walmsley, C. M., Pineau des Forets, G., \& Flower, D. R. 1997, A\&A, 321, 293

Schisano, E., Rygl, K. L. J., Molinari, S., et al. 2014, ApJ, 791, 27

Schneider, N., Bontemps, S., Simon, R., et al. 2011, A\&A, 529, A1

Schneider, N., Csengeri, T., Hennemann, M., et al. 2012, A\&A, 540, L11

Schneider, N., Bontemps, S., Motte, F., et al. 2016, A\&A, 591, A40

Smith, R. J., Glover, S. C. O., Klessen, R. S., \& Fuller, G. A. 2016, MNRAS, 455,3640

Szűcs, L., Glover, S. C. O., \& Klessen, R. S. 2014, MNRAS, 445, 4055

Tielens, A. G. G. M., \& Hollenbach, D. 1985, ApJ, 291, 722

Tritsis, A., \& Tassis, K. 2018, Science, 360, 635

van der Tak, F. F. S., Black, J. H., Schöier, F. L., Jansen, D. J., \& van Dishoeck, E. F. 2007, A\&A, 468, 627

Vázquez-Semadeni, E., Palau, A., Ballesteros-Paredes, J., Gómez, G. C., \& Zamora-Avilés, M. 2019, MNRAS, 490, 3061

Vilas-Boas, J. W. S., Myers, P. C., \& Fuller, G. A. 1994, ApJ, 433, 96

Visser, R., van Dishoeck, E. F., \& Black, J. H. 2009, A\&A, 503, 323

Wenger, M., Ochsenbein, F., Egret, D., et al. 2000, A\&AS, 143, 9

Whitworth, A. P., \& Jaffa, S. E. 2018, A\&A, 611, A20 


\section{Appendix A: SOFIA [ $\left.\mathrm{O}_{1}\right]$ and $\left[\mathrm{C}_{\|}\right]$observations}
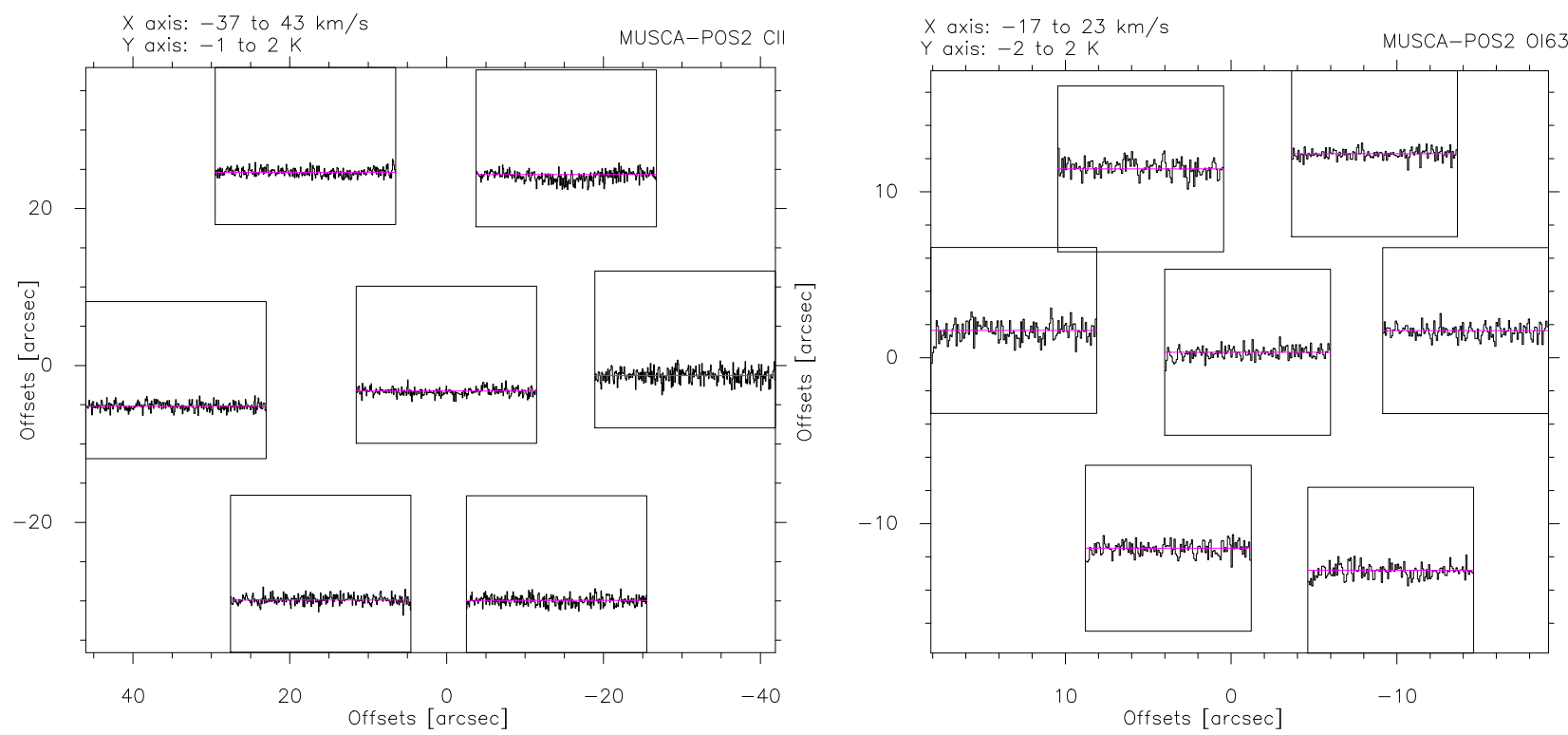

Fig. A.1. Left: observed pixels for the [C II] $158 \mu \mathrm{m}$ line with the SOFIA telescope with a spectral resolution of $0.3 \mathrm{~km} \mathrm{~s}^{-1}$. Right: observed pixels for the [O I] $63 \mu \mathrm{m}$ line.

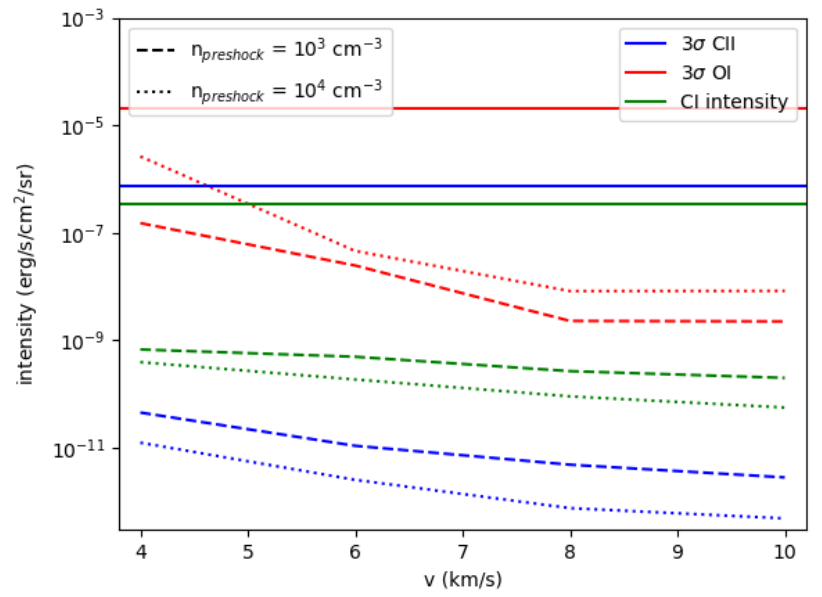

Fig. A.2. Intensity of [C II] (blue), [O I] (red), and [C I] (green) predicted for J-type shock models with a preshock density of $n=10^{3} \mathrm{~cm}^{-3}$ and $n=10^{4} \mathrm{~cm}^{-3}$ by the Paris-Durham code. The horizontal lines indicate the observed [C $\mathrm{I}]$ intensity and $3 \sigma$ upper limits for [C II] and [O I]. This shows that the $[\mathrm{CII}]$ and $[\mathrm{OI}]$ non-detections in Musca are consistent with shocks and that the $[\mathrm{C} \mathrm{I}]$ brightness from the shock is not brighter than the total observed [ $\mathrm{C}$ I] brightness in Musca.

The individual pixels of the upGREAT LFA and HFA, covering the [C $\mathrm{II}] 158 \mu \mathrm{m}$ line and the [O I] $63 \mu \mathrm{m}$ line, respectively, are displayed in Fig. A.1. Both lines, $\left[\mathrm{C}_{\mathrm{II}}\right]$ and $\left[\mathrm{O}_{\mathrm{I}}\right]$ are not detected above an rms (depending on the pixel) of 0.06 and $0.13 \mathrm{~K}$ for the LFA, and 0.11 and $0.21 \mathrm{~K}$ for the HFA.

$\left[\mathrm{O}_{\mathrm{I}}\right]$ is a possible shock tracer, but its non detection does not argue against a possible shock excitation, see Fig. A.2, because of the difficulty to excite [O I] emission. It either needs highvelocity shocks (Hollenbach \& McKee 1989), an irradiated lower velocity shock (Lesaffre et al. 2013; Godard et al. 2019) or dense J/CJ-type shocks (Flower \& Pineau Des Forêts 2010; Gusdorf et al. 2017). Simulations of massive molecular cloud formation through colliding flows predict an $\left[\mathrm{O}_{\mathrm{I}}\right]$ brightness around
0.01-0.05 $\mathrm{K} \mathrm{km} \mathrm{s}^{-1}$ (Bisbas et al. 2017). Yet, such predicted brightnesses are highly uncertain because of the unconstrained atomic oxygen abundance in the ISM (e.g. Meyer et al. 1998; Cartledge et al. 2004; Jenkins 2009; Hincelin et al. 2011).

\section{Appendix B: A two layer multi-component model}

To investigate possible effects of self-absorption, we apply a multi-component dual layer model (Guevara et al. 2020) to the observations of the $\mathrm{CO}(2-1)$ isotopologues. This LTE model fits the radiative transfer equations for multiple components distributed in two layers. The assumption of LTE for low-J CO lines is a justified approximation, as discussed in for example Goldsmith \& Langer (1999); Liszt et al. (2006). van der Tak et al. (2007) also show that the LTE model is mostly valid for the ground state $\mathrm{CO}(1-0)$ transition and deviations from this approximation increase with the quantum number of the upper level. Here, we only use the $\mathrm{CO}(2-1)$ isotologue transitions. The fitted radiative transfer equation is given by

$$
\begin{aligned}
T_{\mathrm{mb}}(\mathrm{v})= & {\left[\sum_{i_{\mathrm{bg}}} \mathcal{J}_{v}\left(T_{\mathrm{ex}, i_{\mathrm{bg}}}\right)\left(1-\mathrm{e}^{-\tau_{i_{\mathrm{bg}}}(\mathrm{v})}\right)\right] \mathrm{e}^{-\sum_{i_{\mathrm{fg}}} \tau_{i_{\mathrm{fg}}}(\mathrm{v})} } \\
& +\sum_{i_{\mathrm{fg}}} \mathcal{J}_{v}\left(T_{\mathrm{ex}, i_{\mathrm{fg}}}\right)\left(1-\mathrm{e}^{-\tau_{i_{\mathrm{fg}}}(\mathrm{v})}\right)
\end{aligned}
$$

with $\mathcal{J}_{v}\left(T_{\text {ex }}\right)$ the equivalent brightness temperature of blackbody emission at a temperature $T_{\mathrm{ex}}$ :

$\mathcal{J}_{v}\left(T_{\text {ex }}\right)=\frac{h v}{k_{\mathrm{B}}} \frac{1}{\mathrm{e}^{T_{0} / T_{\text {ex }}-1}}$,

with the equivalent temperature of the excited level $T_{0}=h v / k_{\mathrm{B}}$ and $v$ the transition frequency. The single components of the model are represented by Gaussian profiles, thus the velocity dependent optical depth of every component is given by:

$\tau(v)=\tau_{0} \mathrm{e}^{-4 \ln 2\left(\frac{v-v_{0}}{w}\right)^{2}}$, 

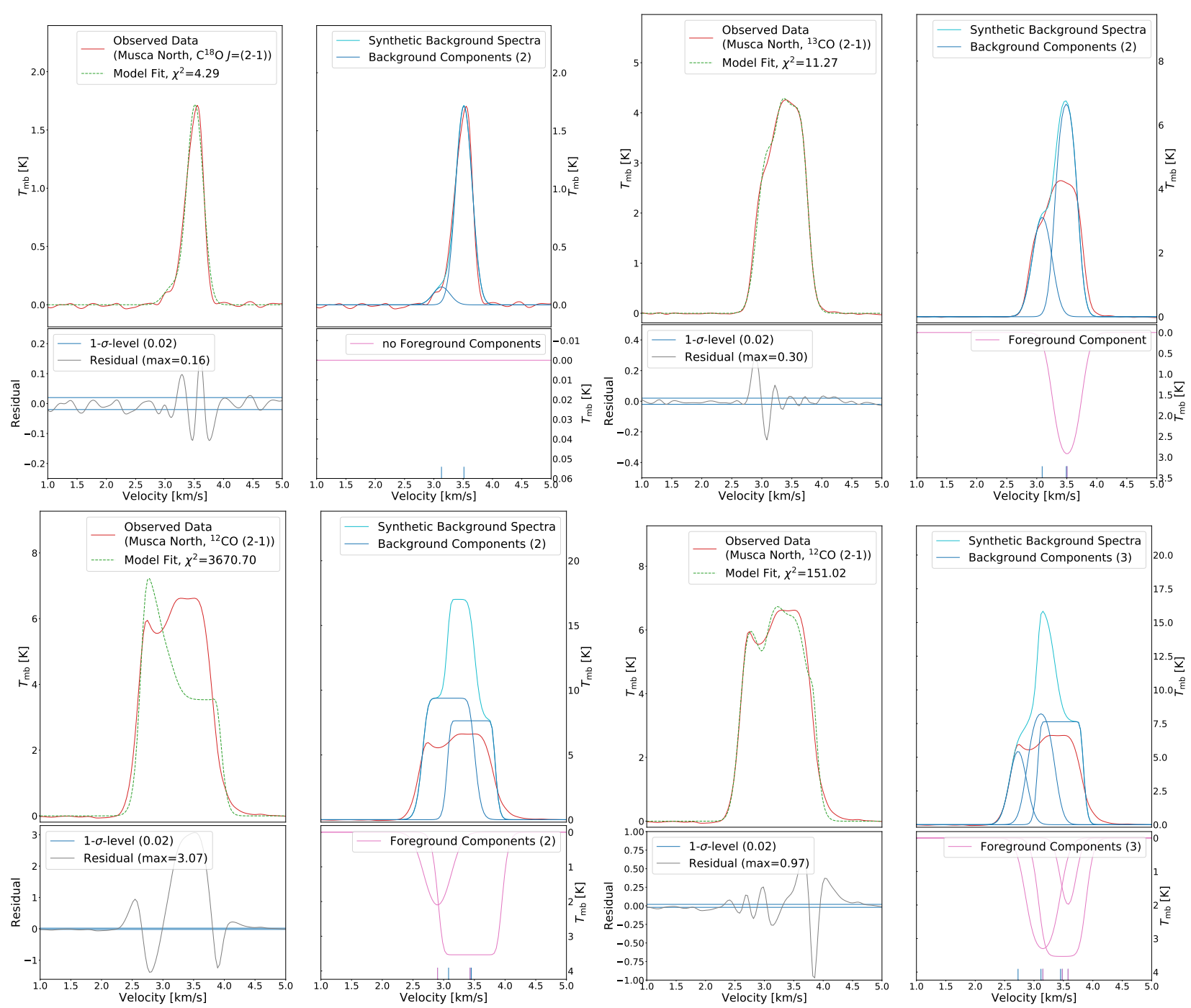

Fig. B.1. Results from a two-layer, multi-component fit to the observed $\mathrm{CO}(2-1)$ isotopes at the northern Musca position. Upper two panels and the lower left panel: fit with two velocity components derived from the optically thin $\mathrm{C}^{18} \mathrm{O}(2-1)$ emission line, lower right panel: best model fit for the observed ${ }^{12} \mathrm{CO}(2-1)$ line. In each panel, upper left sub-panel: observed data as a red line and the model fit as a green dashed line. Upper right sub-panel: individual components, lower left sub-panel: residuals and lower right sub-panel: information on the number and contribution of the foreground. It becomes obvious that two components can be fitted for $\mathrm{C}^{18} \mathrm{O}(2-1)$ and ${ }^{13} \mathrm{CO}(2-1)$, but are insufficient for the ${ }^{12} \mathrm{CO}(2-1)$ line.

with $\mathrm{v}_{0}$ the central (LSR) velocity of the component and $w$ the line width (FWHM).

Each of our components (background and foreground alike) are characterized by four quantities: excitation temperature, optical depth, position (LSR velocity), and width (FWHM). For a given number of components the model fit converges towards the best values for each parameter in a given range. The line position and its width are confined by the observed line profile, thus the excitation temperature and optical depth remain free parameter. The combination of $T_{\mathrm{ex}}$ and $\tau$ is degenerate, that is, higher excitation temperatures go together with lower optical depths and vice versa (and then give a similar fit). However, we can to first order constrain the gas temperature using the Herschel dust temperature $T_{d}$.

The Musca filament (in particular in the crest), is most likely heated by cosmic rays because neither thermal (low densities) nor radiative heating (low FUV field) dominates. The main cooling of the gas is happening via the low-J CO lines. Because of the low densities, freeze-out of $\mathrm{CO}$ is probably not so significant as it is for high densities. Standard dust models (Goldsmith 2001) predict a difference between gas and dust temperature (with $\mathrm{T}_{\mathrm{g}}>\mathrm{T}_{\mathrm{d}}$ ), while recent studies (Ivlev et al. 2019), considering also the grain size distribution, anticipate a less strong difference between gas and dust temperatures. We thus use as a starting value the dust temperatures derived from Herschel shown in Fig. 4 and apply a gas excitation temperature in the range of 10 to $13 \mathrm{~K}$ for the filament crest component and of $\mathrm{T}>13 \mathrm{~K}$ for the shoulder+blueshifted component. Therefore, only the optical depth remains as a free parameter.

The two layer multi-component model is applied to the northern position (the results for the southern position are similar) iteratively for the three transition lines: $\mathrm{C}^{18} \mathrm{O}(2-1)$, ${ }^{13} \mathrm{CO}(2-1)$ and ${ }^{12} \mathrm{CO}(2-1)$, as shown in Fig. B.1.

We start with the $\mathrm{C}^{18} \mathrm{O}(2-1)$ line where we expect no absorption due to the foreground. This way we determine the background components, detected with this line, which are simply scaled up by the natural abundance ratio for the two remaining 
Table B.1. Model results for the two layer multi-component fit.

\begin{tabular}{|c|c|c|c|c|c|c|c|c|}
\hline \multirow[b]{2}{*}{$\begin{array}{c}\text { Fit parameter } \\
\text { Units }\end{array}$} & \multicolumn{4}{|c|}{ Background } & \multicolumn{4}{|c|}{ Foreground } \\
\hline & $\begin{array}{l}T_{\mathrm{ex}} \\
(\mathrm{K})\end{array}$ & $\tau$ & $\begin{array}{c}v \\
\left(\mathrm{~km} \mathrm{~s}^{-1}\right)\end{array}$ & $\begin{array}{c}\Delta v \\
\left(\mathrm{~km} \mathrm{~s}^{-1}\right)\end{array}$ & $\begin{array}{l}T_{\mathrm{ex}} \\
(\mathrm{K})\end{array}$ & $\tau$ & $\begin{array}{c}v \\
\left(\mathrm{~km} \mathrm{~s}^{-1}\right)\end{array}$ & $\begin{array}{c}\Delta v \\
\left(\mathrm{~km} \mathrm{~s}^{-1}\right)\end{array}$ \\
\hline $\mathrm{C}^{18} \mathrm{O}$ component 1 & 12.50 & 0.24 & 3.51 & 0.31 & - & - & - & - \\
\hline $\mathrm{C}^{18} \mathrm{O}$ component 2 & 14.00 & 0.02 & 3.13 & 0.34 & - & - & - & - \\
\hline${ }^{13} \mathrm{CO}$ component 1 & 12.70 & 1.70 & 3.49 & 0.31 & 7.69 & 1.70 & 3.50 & 0.39 \\
\hline${ }^{13} \mathrm{CO}$ component 2 & 13.80 & 0.41 & 3.09 & 0.35 & - & - & - & - \\
\hline${ }^{12} \mathrm{CO}$ component 1 & 12.35 & 90.00 & 3.45 & 0.30 & 7.80 & 7.90 & 3.48 & 0.44 \\
\hline${ }^{12} \mathrm{CO}$ component 2 & 14.80 & 1.75 & 3.12 & 0.37 & 8.13 & 2.00 & 3.15 & 0.45 \\
\hline${ }^{12} \mathrm{CO}$ component 3 & 69.00 & 0.09 & 2.73 & 0.34 & 10.00 & 0.45 & 3.58 & 0.28 \\
\hline
\end{tabular}
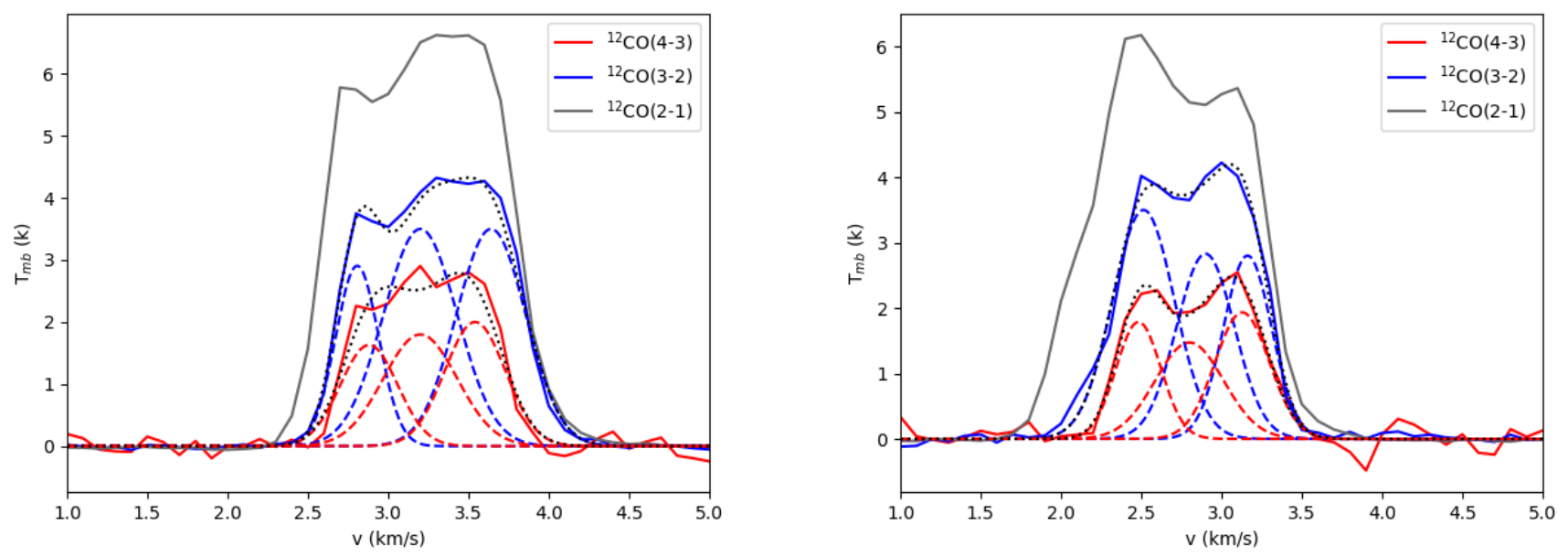

Fig. B.2. Results of a fit of three Gaussian profiles to the ${ }^{12} \mathrm{CO}(3-2)$ and ${ }^{12} \mathrm{CO}(4-3)$ lines towards the crest in the northern map (left) and southern map (right). The solid lines are the observed data, the long-dashed lines the fit with three individual components, and the short-dashed lines the resulting spectrum.

molecular carbon lines. The physical parameter of each component obtained from the model fit are summarized in Table B.1 and plotted in the upper left panel of Fig. B.1. This fit constrains the position and optical depth of the background component.

We then continue to model the ${ }^{13} \mathrm{CO}(2-1)$ line profile. Because the background components are here constrained by the $\mathrm{C}^{18} \mathrm{O}(2-1)$ fit, we scale up the background components by the ${ }^{13} \mathrm{CO} / \mathrm{C}^{18} \mathrm{O} \sim 7$ ratio. We do not strictly fix the parameter obtained from the $\mathrm{C}^{18} \mathrm{O}(2-1)$ model fit, but allow the model to find the best fit in a small range around the given parameter.

The scaled up background emission overshoots the observed line at $v \sim 3.5 \mathrm{~km} \mathrm{~s}^{-1}$. To model the observed line intensities, we add a cold foreground layer with $T<10$. The foreground component absorbs the overshooting emission, see the upper right panel of Fig. B.1. Note, however, that it is unlikely that we have a cooler layer that "wraps around" the filament (which we consider a cylinder) because the Herschel temperature map clearly shows an increasing temperature when going outwards from the crest, see Fig. 4. The only possible way that one could have cool foreground emission that can absorb emission, is by considering a clumpy medium that is unresolved by the Herschel beam. In this unresolved clumpy medium, located in the foreground of the line of sight, one then would need mixed relatively cold $(<10 \mathrm{~K})$ and warm gas $(>15 \mathrm{~K})$ which gives rise to a steadily increasing average temperature in the Herschel beam when moving outwards from the filament crest.
The second blueshifted component only matches the observed emission with an increased ${ }^{13} \mathrm{CO} / \mathrm{C}^{18} \mathrm{O}$ abundance ratio, see Table B.1. The abundance ratio between the molecules is not a very well defined value. The processes of selective photodissociation and carbon isotopic fractionation can modify the relative abundances of CO isotopologues (e.g. Visser et al. 2009; Liszt 2017). In particular in more diffuse regions, the fractionation via the exchange reaction between ${ }^{13} \mathrm{C}^{+}$and ${ }^{12} \mathrm{CO}$ leads to an enhancement of the ${ }^{13} \mathrm{CO}$ abundance (e.g. Liszt \& Ziurys 2012; Röllig \& Ossenkopf 2013; Szúcs et al. 2014). In addition - though probably less important for Musca because of the low FUV field - the more abundant ${ }^{12} \mathrm{CO}$ and ${ }^{13} \mathrm{CO}$ isotopologues shield themselves from the destructive effect of FUV photons more efficiently than the less abundant $\mathrm{C}^{18} \mathrm{O}$ isotopologue because the photodissociation of $\mathrm{CO}$ is governed by line absorption. In this context we note that Hacar et al. (2016) found indications of strong fractionation leading to a significant increase of the ${ }^{13} \mathrm{CO} / \mathrm{C}^{18} \mathrm{O}$ abundance ratio above the standard value of 7 at $A_{\mathrm{V}}<3$. Further analysis of this abundance ratio in our companion paper on the Musca filament confirms this result presented in Hacar et al. (2016).

In the previous two model fits, we determined the background and foreground components which we now scale up by the abundance ratio ${ }^{12} \mathrm{CO} /{ }^{13} \mathrm{CO} \sim 60$. Note that this value can be significantly lower because of fractionation (see above). The resulting fit is shown in the left lower panel of Fig. B.1. 
Due to the large optical depths, we observe flat-topped spectra in the background and foreground layer. Since the observed intensities do not fit the natural carbon abundance ratio for the second component we only fix the first component and allowed the model to vary over a range of values in the second component to find the best possible solution. In addition we included a foreground component, which would only be observable in the ${ }^{12} \mathrm{CO}$ isotope, to remove the emission excess in the blueshifted wing and possibly reproduce the bump located in the blueshifted part of the observed ${ }^{12} \mathrm{CO}$ spectrum. The resulting spectra show a flat spectrum at velocities with bright $\mathrm{C}^{18} \mathrm{O}(2-1)$ emission, followed by an emission peak at the blue-shifted wing. Thus, the model results do not manage to represent the line shape, the observed bump in particular, and intensity by taking selfor foreground absorption effects of the scaled up background components derived from the isotopes, see lower left panel of Fig. B.1.

Since two background components do not represent the observed spectrum, we include an additional component to the model, which is only visible in the ${ }^{12} \mathrm{CO}$ spectrum but vanishes in the noise for the weaker isotopes, see lower right panel of Fig. B.1. To meet this criteria we need to include a background component with low optical depth, which results in an increased temperature since a low optical depth requires an increase in temperature to produce the same amount of emission. This third background component is located at $v=2.8 \mathrm{~km} \mathrm{~s}^{-1}$, see Table B.1. In addition we allow cold optically thin components in order to correct for the emission excess in the central and red-shifted part of the spectrum. As in the previous model fit we do not fix the central second component by the carbon abundance ratio, but allow it to vary in a wide range to find the best model fit to the observed data. We thus manage to represent the spectrum well, only with three background components.

\section{Appendix C: SiO non-detection}

The observations with the PI230 instrument on APEX towards the Musca cloud presented in Paper I covered the $\mathrm{SiO}(5-4)$ line. We have presented the detection of warm gas heated by low-velocity shocks, which makes the observation of $\mathrm{SiO}(5-4)$ interesting since some molecular clouds display extended $\mathrm{SiO}$ emission with relatively narrow linewidths (Jiménez-Serra et al. 2010; Nguyen-Lu'o'ng et al. 2013; Duarte-Cabral et al. 2014). This suggests that this is not $\mathrm{SiO}$ sputtered from the grains by protostellar outflows (e.g. Schilke et al. 1997; Gusdorf et al. 2008a). To explain this extended $\mathrm{SiO}$ emission in W43, it was considered that $1-10 \%$ of $\mathrm{Si}$ was not locked in the grains in the preshock gas (Louvet et al. 2016).

In Musca, because of the low-velocity shocks, sputtering of $\mathrm{SiO}$ from the grains seems improbable (Gusdorf et al. 2008b;
Louvet et al. 2016). Upon analysing the data after averaging over the filament crest $\left(N>3 \times 10^{21} \mathrm{~cm}^{-2}\right)$, no $\mathrm{SiO}$ emission is detected with an rms of $0.0134 \mathrm{~K}$.

Since $\mathrm{SiO}$ has a high critical density $\left(>10^{6} \mathrm{~cm}^{-3}\right)$ it requires a non-LTE approach (Csengeri et al. 2016) to study the maximal $\mathrm{SiO}$ abundance in the gas phase. Therefore, we use RADEX to search for an upper limit on the $\mathrm{SiO}$ column density. This is done for two densities $10^{3}$ and $10^{4} \mathrm{~cm}^{-3}$, assuming a minimal temperature of $10 \mathrm{~K}$ and a FWHM of $0.5 \mathrm{~km} \mathrm{~s}^{-1}$ (similar to the $\mathrm{C}^{18} \mathrm{O}$ linewidth). The resulting $3 \sigma \mathrm{SiO}$ column density upper limits are $\sim 10^{14}$ and $10^{15} \mathrm{~cm}^{-2}$ for densities of $10^{4}$ and $10^{3} \mathrm{~cm}^{-3}$ respectively.

This upper limit is observed toward the filament crest, which has a Herschel dust column density of $\sim 4 \times 10^{21} \mathrm{~cm}^{-2}$, such that the upper limit obtained at a density of $10^{4} \mathrm{~cm}^{-3}$ should hold, see Paper I. Assuming all hydrogen is in molecular form, using $[\mathrm{Si}] /[\mathrm{H}]=2 \times 10^{-5}$ and taking that all $\mathrm{Si}$ is found in the form of $\mathrm{SiO}$ (Louvet et al. 2016). This poses an upper limit of $0.06 \%$ on the $\mathrm{Si}$ abundance in the gas phase $\left(0.6 \%\right.$ when assuming $n_{\mathrm{H}_{2}}=$ $10^{3} \mathrm{~cm}^{-3}$ ).

This indicates that in a molecular cloud at an early stage of evolution basically all $\mathrm{Si}$ is locked in the grains and that one needs environments with a radiative or dynamic history like W43 or protostellar outflows to release $\mathrm{SiO}$ in the gas phase.

\section{Appendix D: Mass accretion rate}

As we have presented the detection of a filament accretion shock, it is possible to estimate the mass accretion rate as a result of this shock using $2 \pi R \cdot \Sigma_{\text {shock }} \cdot t_{\mathrm{psc}}^{-1}$. Where $\mathrm{R}$ is the radius that encompasses most dense gas $(\approx 0.2 \mathrm{pc}), \Sigma_{\text {shock }}$ is the column density of the shock layer, and $t_{\mathrm{psc}}$ is the post-shock cooling time for which we use the expression in Whitworth \& Jaffa (2018)

$t_{\mathrm{psc}} \lesssim 1.2 \mathrm{Myr}\left(\frac{X_{\mathrm{CO}}}{3 \cdot 10^{-4}}\right)^{-1}\left(\frac{n_{\mathrm{H}_{2}, \mathrm{O}}}{\mathrm{cm}^{-3}}\right)^{-1}\left(\frac{\mathrm{v}_{\mathrm{s}}}{\mathrm{kms}^{-1}}\right)^{-1}$,

with $n_{\mathrm{H}_{2}, 0}$ the pre-shock density and $v_{\mathrm{s}}$ the shock velocity.

To calculate $\Sigma_{\text {shock }}$ we start from the $\Sigma_{12}$ CO column density of the RADEX models that manage to reach the observed ratios in Sect. 3.5, which is $9 \times 10^{14} \mathrm{~cm}^{-2} . \Sigma_{\text {shock }}$ is then given by $\Sigma_{{ }^{12} \mathrm{CO}} / \mathrm{X}_{\mathrm{CO}}$. This $\mathrm{X}_{\mathrm{CO}}$ cancels out with the same term in Eq. (D.1).

In Eq. (D.1), we use an estimated preshock density $n_{\mathrm{H}_{2}, 0}$ $=4 \times 10^{2} \mathrm{~cm}^{-3}, v_{\mathrm{s}}=0.9 \mathrm{~km} \mathrm{~s}^{-1}$ and assume that the equality in Eq. (D.1) roughly holds. This implies that our mass accretion rate estimate here will be a lower limit. Putting all this together results in an estimated minimal mass accretion rate of $20 M_{\odot} \mathrm{pc}^{-1} \mathrm{Myr}^{-1}$. 